\title{
Discrete effects on the forcing term for the lattice Boltzmann modeling of steady hydrodynamics
}

\author{
Goncalo Silva \\ LAETA, IDMEC, Mechanical Engineering Department, IST, University of Lisbon, Lisbon 1049-001, Portugal
}

\section{A R T I C L E I N F O}

\section{Article history:}

Received 14 January 2020

Revised 27 March 2020

Accepted 15 April 2020

Available online 19 April 2020

\section{MSC:}

00-01

99-00

\section{Keywords:}

Lattice Boltzmann method

Two-relaxation-time scheme

Analytical solutions

Discrete error analysis

Non-uniform body forces

\begin{abstract}
A B S T R A C T
This work concerns with the clean inclusion of the forcing term in the lattice Boltzmann method (LBM) for the modeling of non-uniform body forces in steady hydrodynamics. The study is conducted for the two-relaxation-time (TRT) scheme. Here, we consider a simple, but yet sufficiently generic, flow configuration driven by a spatially varying body force based on which we derive the analytical solution of the forced LBM-TRT and compare two force strategies set by first- and second-order expansions. This procedure exactly establishes the macroscopic system satisfied by each force formulation at discrete level. The obtained theoretical results are further verified in two distinct benchmark channel flow problems. Overall, this study shows that the spatial discrete effects posed by the LBM modeling of the force term may come in through two sources. The first one is a defect inherent to LBM, arising from the non-local spatial discretization of the forcing term, and given by the discrete Laplacian of the body force. While it corrupts the discrete momentum balance with a non-linear viscosity dependent term in the single-relaxation-time schemes, this inconsistency is avoided with the TRT scheme, through its free-tunable relaxation degree of freedom $\Lambda$. The other error source is unique to the use of second-order force expansions, where its nonzero second-order velocity moment interferes with the discrete momentum balance through a spurious first-order derivative term, leading to several forms of inconsistency in the LBM steady solution. For simulations under the convective scaling it makes the LBM scheme a numerical representation of a differential system distinct from the physical one, whereas under the diffusive scaling it leads to viscosity-dependent numerical errors, which corrupt the otherwise consistent structure of TRT steady-state solutions. In contrast, the defects reported herein are absent with the first-order force expansion scheme operated within the scope of the LBM-TRT model for steady hydrodynamics.
\end{abstract}

(C) 2020 Elsevier Ltd. All rights reserved.

\section{Introduction}

The lattice Boltzmann method (LBM) [1-3] is a well established numerical technique to solve the Navier-Stokes equations (NSE). Despite its many inherent advantages, cf. the review articles [4,5] and the monographes [6,7], the crucial step to bridge the link between the mesoscopic LBM formulation and the specific hydrodynamics variables still presents some challenges. For the "athermal" LBM, such a consistency link is expressed by the simultaneous satisfaction of the following two points: (i) the LBM first three velocity moments should match the required hydrodynamic expressions and (ii) no numerical artifacts should be introduced during the discretisation process.

\footnotetext{
Fully documented templates are available in the elsarticle package on CTAN. E-mail address: goncalo.nuno.silva@gmail.com
}

The present work deals with the implementation of external body forces in LBM, a topic with both theoretical and applied interest, e.g., in multiphase flows [8-11], flows in porous media [1215], rotating fluid flows [16-19], turbulent flows [20-23], etc. In this regard, it is well-established that, when translated into the formulation of forces, each of the two consistency points mentioned above relates to a specific discretization step. Point (i), concerned with the proper choice of velocity moments, is dictated by the velocity expansion order of the forcing term [24-26,31], whereas point (ii), concerned with the reduction of discrete lattice artifacts, is favoured with the second-order space-time discretization of the forcing term [27-31]. Although the superiority of the second-order space-time discretization is undisputed, it should be noted that inherent discrete force errors still exist. This is documented in $[11,14,32,33,36]$ and will be further pointed out in this work. Yet, the more controversial issue lies in the order of the velocity space expansion of the LBM forcing term where some works, 
e.g., [24,25,30,31], advocate the inclusion of second-order terms while others, e.g., $[14,27,29,37,40,41]$, prefer the first-order truncation. The correct force expansion order for steady flow simulations will be the main topic of this work.

Early works $[38,39]$ have already discussed this issue, attributing to this source of controversy the lack of distinction between steady- and unsteady-state dynamics in the numerical model formulation. This distinction is well-established within CFD community. For example, the numerical diffusion phenomenon, which originates from a mixed space/time derivative term of the advective flux, is known to affect time-dependent problems, where it can be cancelled with the inclusion of a anti-numerical-diffusion correction [42-45]. At steady-state this error does not exist, so the inclusion of such a correction, rather than beneficial, will introduce the exact same numerical diffusion artefact that was designed to eliminate in the first place. A very similar explanation applies to the duality in the truncation order of the LBM forcing term. In time-dependent hydrodynamic problems there is a spurious viscous stress term, given by a mixed space/time derivative of the Euler momentum flux, which introduces a body force artefact, as independently noted by many studies $[24,30,31,39]$. The inclusion of the second-order velocity term in the force expansion views the elimination of this body force artefact, mimicking the role of the anti-numerical-diffusion correction mentioned above. That is why, when applied to steady problems, the inclusion of this secondorder force term does not meet a similar term to cancel, rather introducing the same artefact that was proposed to eliminate.

These conclusions reported above correspond to the original findings of $[38,39]$ and were established recurring to the traditional second-order Chapman-Enskog expansion [1,4,7,37] applied to both time-independent and time-dependent settings; this latter subject to either convective or diffusive scalings. The work [39] showed that only the time-dependent setting, when accessed through the convective scaling, is compatible with the secondorder force expansion, while the remaining cases should stick with a first-order force scheme. These observations back up previous studies that, by adopting the diffusive scaling under distinct theoretical frameworks, e.g. the Chapman-Enskog expansion in [37] or the (Hilbert) regular asymptotic expansion in [41], agreed on the first-order force expansion as the correct choice to reproduce the hydrodynamic external force in the time-dependent incompressible Navier-Stokes equations.

The present study aims to re-evaluate the aforementioned results by focusing on the time-independent setting, i.e. the less consensual case since the steady-state Chapman-Enskog analysis undertaken in $[38,39]$ remains less popular. Thus, with the modeling of steady hydrodynamics in mind, the purpose of this work is to examine the possible sources of spatially-dependent force errors based on an exact analysis. For that, we study a simple, but yet sufficiently generic, flow configuration of an incompressible fluid driven by a spatially varying body force, which permits deriving the analytical solution of the forced LBM scheme following the procedures of He et al. [46] and Ginzburg and d'Humi Âlires [14,47]. The two techniques are presented herein for their pedagogical interest, as they lead to identical results; our goal is to show that the procedure of $[14,47]$, although less popular, is considerably simpler than [46]. With the adopted theoretical procedures we divert from the scope of approximate perturbative methods, e.g. [29,30,39], and exactly establish the consistency of each force scheme with respect to steady hydrodynamics in an unambiguous way, where possible force error sources are identified at discrete level. The theoretical study is complemented with numerical tests where the effect of the force errors is assessed from the perspective of both convective and diffusive scalings. This will prove useful in understanding the role of the identified errors and how they impact the correctness of simulations.
In this work, we consider the two-relaxation-time (TRT) LBM scheme [37], which is presented in Section 2 together with the inspected forcing schemes. They are theoretically studied in Section 3, where we derive the exact solution of the forced LBMTRT scheme for the aforementioned steady-state flow configuration, extending previous works, e.g., $[10,13,14,48]$, to a non-uniform force-driven setup; the details of the underlying derivations are shown in Appendix A and Appendix B. Then, in Section 4, the exact results are verified for two benchmark flow problems, each possessing known analytical solution; they model: (i) the porous channel flow with wall injection and (ii) the rotating channel Poiseuille flow. Finally, the main conclusions of this study are summarized in Section 5.

\section{LBM-TRT Model}

The LBM [6,7] solves for the populations $f_{q}(\vec{x}, t)$, defined on space $\vec{x}$ and time $t$, along a discrete velocity set, called lattice, featuring one immobile $\overrightarrow{c_{0}}=\overrightarrow{0}$ and $Q-1$ non-zero velocity vectors $\vec{c}_{q}$ per grid node. The LBM evolves through a succession of streaming and collision steps. In this work the collision step adopts the two-relaxation-time (TRT) model [37]. The TRT is formulated on the symmetry argument that any lattice quantity $\psi_{q}$ can be decomposed onto symmetric $\psi_{q}^{+}=\frac{1}{2}\left(\psi_{q}+\psi_{\bar{q}}\right)$ and anti-symmetric $\psi_{q}^{-}=\frac{1}{2}\left(\psi_{q}-\psi_{\bar{q}}\right)$ components, where $\vec{c}_{\bar{q}}=-\vec{c}_{q}$. On this basis, the evolution equation of LBM-TRT, with an external source term $S_{q}$ considered explicitly, reads as follows [37]:

$$
\begin{aligned}
f_{q}\left(\vec{x}+\vec{c}_{q} \delta t, t+\delta t\right)= & {\left[f_{q}-\frac{1}{\tau^{+}}\left(f_{q}^{+}-e_{q}^{+}\right)-\frac{1}{\tau^{-}}\left(f_{q}^{-}-e_{q}^{-}\right)\right.} \\
& \left.+\left(1-\frac{1}{2 \tau^{+}}\right) S_{q}^{+} \delta t+\left(1-\frac{1}{2 \tau^{-}}\right) S_{q}^{-} \delta t\right] \\
& \times(\vec{x}, t) .
\end{aligned}
$$

By design the collision model in TRT is controlled by two relaxation times: $\tau^{+}$that controls symmetric modes and sets the fluid viscosity $\nu$, and $\tau^{-}$that controls anti-symmetric modes and is a free parameter. Most importantly, the function formed by these two relaxations $\Lambda=\left(\tau^{+}-\frac{1}{2}\right)\left(\tau^{-}-\frac{1}{2}\right)$ is the only trace of the relaxation parameters pre-factoring the spatial truncation errors of TRT. Thus, $\Lambda$ is what controls the stationary field of nondimensional TRT solutions [14,37,47]. The Navier-Stokes equations (NSE) are modelled with the following $e_{q}^{-}$(anti-symmetric) and $e_{q}^{+}$ (symmetric) equilibrium [37]:

$e_{q}^{-}=t_{q}^{\star} \frac{c_{q \alpha} \bar{\rho} u_{\alpha}}{c^{2}}, \quad e_{q}^{+}=t_{q}^{\star}\left[\frac{P}{c^{2}}+\left(3 c_{q \alpha} c_{q \beta}-c^{2} \delta_{\alpha \beta}\right) \frac{\bar{\rho} u_{\alpha} u_{\beta}}{2 c^{4}}\right]$.

Above, $u_{\alpha}$ is the fluid velocity and $P$ is the pressure, related to density $\rho$ by the equation of state $P=c_{s}^{2} \rho$, where the lattice sound speed $c_{s}$ is a free parameter, limited by the necessary stability condition $1-c_{s}^{2} \sum_{q=0}^{Q-1} t_{q}^{\star}>0$ [44]. The density ground state is $\bar{\rho}:=\rho_{0}$, with $\rho_{0}=$ const., according to the incompressible model [49] (for the compressible case $\bar{\rho}:=\rho(\vec{x}, t)$, but this case is not considered herein). The inclusion of an external acceleration field $\vec{a}$ is expressed by the standard LBM forcing term given by:

$$
\begin{gathered}
S_{q}^{-}=t_{q}^{\star} \frac{c_{q \alpha} \bar{\rho} a_{\alpha}}{c^{2}}, \quad S_{q}^{+}=\mathcal{A} t_{q}^{\star}\left[\left(3 c_{q \alpha} c_{q \beta}-c^{2} \delta_{\alpha \beta}\right) \frac{\bar{\rho} a_{\alpha} u_{\beta}}{c^{4}}\right] \\
\text { with } \begin{cases}\mathcal{A}=0 & \text { first - order force expansion, } \\
\mathcal{A}=1 & \text { second - order force expansion. }\end{cases}
\end{gathered}
$$

Note, the symmetric source term $S_{q}^{+}$accounts for two forcing formulations, as determined by $\mathcal{A}$ in Eq. (3). 
The zeroth- and first-order velocity moments of the LBM solution establish the mass density and momentum density:

$\rho=\sum_{q=0}^{Q-1} f_{q}, \quad \bar{\rho} \vec{u}=\sum_{q=1}^{Q-1} \vec{c}_{q} f_{q}+\frac{\delta t}{2} \bar{\rho} \vec{a}$.

The source term, Eq. (3), possesses the following zeroth-, firstand second-order velocity moments:

$$
\begin{aligned}
& \sum_{q=0}^{Q-1} S_{q}^{+}=0, \quad \sum_{q=1}^{Q-1} \vec{c}_{q} S_{q}^{-}=\bar{\rho} \vec{a}, \\
& \sum_{q=1}^{Q-1} c_{q \alpha} c_{q \beta} S_{q}^{+}=\mathcal{A} \bar{\rho}\left(a_{\alpha} u_{\beta}+a_{\beta} u_{\alpha}\right) .
\end{aligned}
$$

\section{Exact analysis of LBM with spatially varying force}

Consider the LBM scheme operating on the two-dimensional nine-velocity (D2Q9) model, defined on the Cartesian system $\vec{x}=(x, y)$, with velocities $\vec{c}_{1}=-\vec{c}_{3}=(1,0) c, \vec{c}_{2}=-\vec{c}_{4}=(0,1) c$, $\vec{c}_{5}=-\vec{c}_{7}=(1,1) c$ and $\vec{c}_{6}=-\vec{c}_{8}=(-1,1) c$. For this model, the weights in the equilibrium, Eq. (2), are $t_{1,2,3,4}^{\star}=1 / 3$ and $t_{5,6,7,8}^{\star}=$ $1 / 12$. Note, the rest population $\overrightarrow{c_{0}}=(0,0) c$, with weight $t_{0}^{\star}=(3-$ $\left.5 c_{s}^{2}\right) / 3 c_{s}^{2}$, is not relevant for the steady-state regime, thus not considered in this work.

In this section, we seek for the analytical solution of the LBMTRT equation, with a spatially-varying external body force, for the macroscopic momentum balance in bulk (i.e. boundary effects are excluded). For the analysis purpose, the following standard conditions are adopted $[10,13,14,48]$ : (1) time-independence; (2) invariance along the $\mathrm{x}$-direction; (3) velocity field $\vec{u}=(u, v)$; and (4) acceleration field $\vec{a}=\left(a_{x}, a_{y}\right)$. The application of conditions (1) and (2) over Eq. (1) provides the following simplified LBM-TRT evolution equation:

$$
\begin{aligned}
f_{q}\left(y+c_{q y} \delta t\right)= & f_{q}(y)-\frac{1}{\tau^{+}}\left(f_{q}^{+}(y)-e_{q}^{+}(y)\right)-\frac{1}{\tau^{-}}\left(f_{q}^{-}(y)-e_{q}^{-}(y)\right) \\
& +\left(1-\frac{1}{2 \tau^{+}}\right) S_{q}^{+}(y) \delta t+\left(1-\frac{1}{2 \tau^{-}}\right) S_{q}^{-}(y) \delta t,
\end{aligned}
$$

where the only non-trivial velocity moment in Eq. (4) computes the $u$ velocity component as follows:

$$
\sum_{q=1}^{Q-1} c_{q x} f_{q}(y)=\bar{\rho} u_{j}-\frac{\delta t}{2} \bar{\rho}\left(a_{x}\right)_{j} .
$$

Hereinafter, the spatial dependency along the $y$ coordinate is expressed in grid units by index $j$, where $y=j \delta x$ and $\delta x=c \delta t$ is the grid space step. By inserting Eq. (6) into Eq. (7), one can analytically solve the exact macroscopic governing equations of the LBM-TRT system. For completeness, this task is shown in Appendix for two theoretically different procedures: He et al. [46] in Appendix A and Ginzburg and d'HumiÂlres [14,47] in Appendix B. Although the former is more popular, the latter is more general and simpler to apply. The most important point for our intents is that, in the end, the two procedures recover the same exact momentum balance for LBM-TRT at discrete level, which is given by:

$$
\begin{aligned}
& \frac{u_{j+1} v_{j+1}-u_{j-1} v_{j-1}}{2 \delta x}-\left(a_{x}\right)_{j}-v \frac{u_{j+1}-2 u_{j}+u_{j-1}}{\delta x^{2}} \\
& +\delta x^{2}\left(\frac{8 \Lambda-3}{12}\right) \frac{\left(a_{x}\right)_{j+1}-2\left(a_{x}\right)_{j}+\left(a_{x}\right)_{j-1}}{\delta x^{2}} \\
& +\mathcal{A} \frac{3 v}{c^{2}}\left(\frac{u_{j+1}\left(a_{y}\right)_{j+1}-u_{j-1}\left(a_{y}\right)_{j-1}}{2 \delta x}+\frac{v_{j+1}\left(a_{x}\right)_{j+1}-v_{j-1}\left(a_{x}\right)_{j-1}}{2 \delta x}\right)=0,
\end{aligned}
$$

where $v=\frac{1}{3} c^{2} \delta t\left(\tau^{+}-\frac{1}{2}\right)$ is the fluid kinematic viscosity.
Eq. (8) corresponds to the second order difference scheme for the continuous differential equation:

$$
\begin{aligned}
& \partial_{y}(u v)-a_{x}-v \partial_{y y} u \\
& +\underbrace{(I) \delta x^{2}\left(\frac{8 \Lambda-3}{12}\right) \partial_{y y} a_{x}}_{\text {Error(I) }}+\underbrace{\mathrm{A} \frac{3 v}{c^{2}}\left(\partial_{y}\left(u a_{y}\right)+\partial_{y}\left(v a_{x}\right)\right)}_{\text {Error(II) }}=0,
\end{aligned}
$$

Eq. (9) brings about two forced-based terms (signalized above) not found in the physical incompressible NSE with an external body force, which is our target differential equation. These two terms have the following meanings:

Error (I) in Eq. (9), represented by the Laplacian of $a_{x}$, is an higher-order correction resulting from the non-local stencil discretization of the (spatially-varying) body force [13,14,48]. This term is intrinsic to the LBM and recognized to impact the LBM modeling of diverse flow problems [14,32]. This correction scales with $\delta x^{2}$, i.e. it is the same order of the LBM discretization error. But though it vanishes in the hydrodynamic limit, i.e. when $\delta x \rightarrow 0$, at finite grid resolutions it holds non-negligible, and even dominant $[13,32,36]$. Additionally, it depends on the relaxation rates. While in TRT it scales with $\Lambda$, in the single-relaxation-time (SRT) collision model [50] this relaxation footprint becomes $\Lambda \propto v^{2}$, which is responsible for creating a non-linear viscosity-dependent artefact $[13,14]$.

Error (II) in Eq. (9), represented by the first-order derivative of the force and velocity product, is not intrinsic to LBM. Rather, it is an artefact created uniquely by the discretization of the second order velocity moment of the force term $[38,39]$, i.e. it is only relevant when $\mathcal{A}=1$ in Eq. (3) and absent when $\mathcal{A}=0$ is considered instead. In practice, the spurious term associated to $\mathcal{A}=1$ leads to an inconsistent numerical scheme, which can be identified in several ways, from the breaking of the TRT viscosity-independent property to the inconsistent convergence towards a differential system distinct from the physical one. These defects will be illustrated in the next section, by examining two test cases.

\section{A note on the effect of the LBM force errors on boundaries}

In flow confined problems the LBM bulk solution, Eq. (8), must be supplemented with the prescription of boundary conditions. However, when a low-order boundary scheme is used, then additional numerical artefacts are likely to be introduced. For example, take a constant force so that Errors (I) and (II) vanish, even here the bounce-back (BB) rule will introduce by itself an error that varies with $\Lambda$ and affects the precision of the no-slip wall location at $\mathcal{O}\left(\delta x^{2}\right)[7,27]$ (i.e. a $\Lambda$-dependent error that affects the $\mathrm{BB}$ accommodation of the flow curvature); this error is particularly harmful in the framework of the SRT collision operator where this $\Lambda$-dependency of BB turns into the well-established $v^{2}$ dependency of the no-slip wall location $[7,27]$. For this horizontal flow setup with halfway walls, this BB error is removed by setting $\Lambda=3 / 16$.

However, a conflict in this $\Lambda$ choice becomes evident when a non-uniform body force is considered. Namely, according to Eq. (8), even when Error (II) is absent due to $\mathcal{A}=0$, the removal of Error (I) will require $\Lambda=3 / 8$ for this setup. Worse, the studies [3234] have identified this problem and showed that Error (I), which is intrinsic to the LBM discretization of non-uniform body forces, gets transferred from bulk to boundaries; for example, in the BB case it leads to body force $\Lambda$-dependent artefacts that affect the precision of the no-slip wall location on a much larger extent, at both $\mathcal{O}(\delta x)$ and $\mathcal{O}\left(\delta x^{2}\right)$ (i.e. they affect the BB accommodation of both the flow gradient and the flow curvature). This implies that, in this case, there is no single $\Lambda$ value capable of fixing the BB wall location at the desired place; for a detailed discussion on the pros 
a)

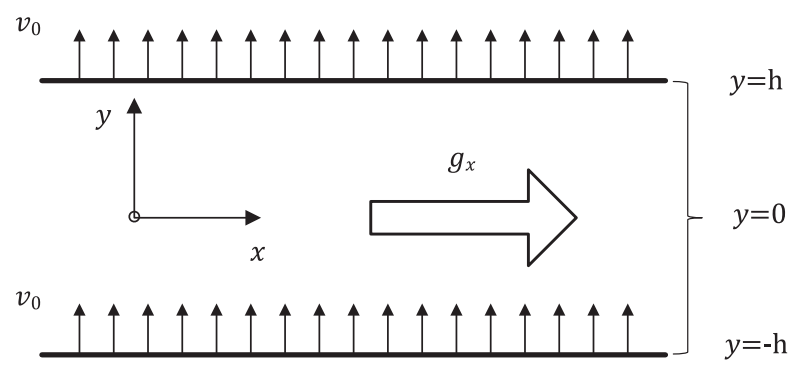

b)

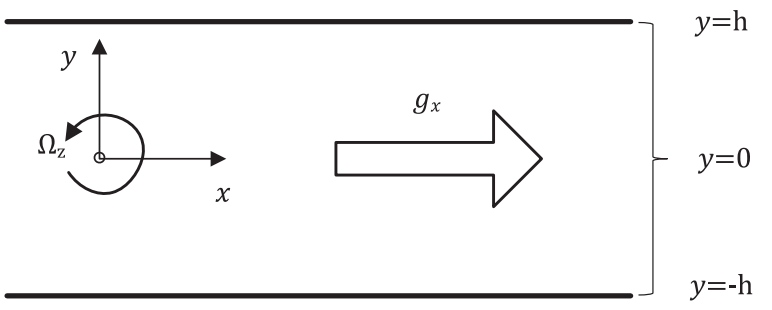

Fig. 1. Schematic representation of studied channel flow problems: (a) porous flow with wall injection, (b) rotating Poiseuille flow.

and cons of using $\Lambda=3 / 16$ vs. $\Lambda=3 / 8$ in the BB wall modeling of this problem class we refer to [32-34].

Two alternative and general strategies to avoid these problems have been devised in [32,33]; specifically, [32] proposed a new bulk force correction while [33] constructed a new class of high-order boundary schemes, both strategies were designed to remove or mitigate the interference of the aforementioned spatiallyvarying force errors in bulk and boundaries, respectively; moreover, they also accounted for the influence of $\Lambda$ on the accuracy of the numerical solutions [33], guaranteeing that $\Lambda$ stays free to optimize other features of the LBM-TRT scheme.

\section{Application of exact analysis and validation}

The analytical solution of the LBM-TRT, previously addressed in Section 3, indicates that the stationary momentum balance of LBM with a force scheme $\mathcal{A}=1$ gives rise to a spurious momentum contribution given by $\partial_{y}\left(u a_{y}+v a_{x}\right)$. The present section is essentially devoted to examine this artefact. For that, we consider the two flow configurations sketched in Fig. 1. They model: (a) porous channel flow with wall injection, and (b) rotating channel Poiseuille flow.

Since the objective of the present study concerns with the bulk flow solution, the numerical tests illustrated herein make use of standard boundary schemes, namely periodic boundary conditions at inlet/outlet boundaries and the bounce-back (BB) rule at solid walls. ${ }^{1}$ The LBM solution is considered at steady-state when $\left|\sum u_{x}(t) / \sum u_{x}\left(t-t_{a}\right)-1\right|<10^{-12}$ with $t_{a}=100 \delta t$. Then, accuracy is measured as $L_{2}(u)=\sqrt{\sum\left(u^{\text {(num })}-u^{\text {(exact) })}\right)^{2} / \sum\left(u^{\text {(exact) })}\right)^{2}}$, with the sums taken over all grid nodes, $u^{(\text {num })}$ the numerical solution and $u^{\text {(exact) }}$ the solution of the continuous problem.

\subsection{Porous channel Brinkman flow with wall injection}

Consider a constant acceleration field $g_{x}$ that drives an incompressible fluid inside a channel filled with a porous medium of homogenous permeability $k$, see Fig. 1 (a). The fluid motion is described by Brinkman's model [12-14,32,33], given by the forcing term:

$a_{x}=g_{x}-\frac{v}{k} u, \quad a_{y}=-\frac{v}{k} v$.

The introduction of Eq. (10) into Eq. (8) establishes the exact discrete momentum balance satisfied by LBM-TRT for this problem,

\footnotetext{
1 To guarantee the LBM results are indeed free from the contamination of boundary effects, we actually considered both BB and Zou-He [51] wall boundary conditions. Based on the observation that conclusions held unchanged, and for the sake of brevity, this paper only shows the BB outcomes.
}

which is given as follows:

$$
\begin{aligned}
& (1+\gamma) \frac{u_{j+1} v_{j+1}-u_{j-1} v_{j-1}}{2 \delta x}-g_{x}+\frac{v}{k} u_{j} \\
& -v_{\text {eff }} \frac{u_{j+1}-2 u_{j}+u_{j-1}}{\delta x^{2}}=0,
\end{aligned}
$$

with coefficients

$\gamma=-\mathcal{A} \frac{6 v^{2}}{k c^{2}}, \quad v_{\text {eff }}=v(1+\overline{\delta v}), \quad \overline{\delta v}=\frac{\delta x^{2}}{k}\left(\frac{8 \Lambda-3}{12}\right)$.

Eq. (11) reproduces the following equivalent differential equation:

$(1+\gamma) \partial_{y}(u v)-g_{x}+\frac{v}{k} u-v_{\text {eff }} \partial_{y y} u=0$

Physically, this problem should be governed by $\partial_{y}(u v)-g_{x}+$ $\frac{v}{k} u-v \partial_{y y} u=0$, which points to two error sources in Eq. (13).

The first one is the $\gamma$ artefact that affects the convective term; $\gamma$ is created by the non-zero second-order velocity moment of the forcing term, due to $\mathcal{A}=1$ (i.e. Error (II) in Eq. (9)), and it only vanishes with a first-order force expansion, where $\mathcal{A}=0$ and therefore $\gamma=0$, see Eq. (12).

The second error source in Eq. (13) refers to the effective viscosity $v_{\text {eff }}$ coefficient, which affects the diffusive term, modifying the actual viscosity value $v$ by an amount equal to $1+\overline{\delta v}$, see Eq. (12). The added artefact $\overline{\delta v}$ is called the viscosity correction [14] and is created by the intrinsic force discretization error (i.e. Error (I) in Eq. (9)). For this problem, $\overline{\delta v}$ depends on: (i) the physical regime, scaling inversely with permeability $k$; (ii) the grid resolution, scaling with the square of mesh resolution $\delta x^{2}$; and (iii) the TRT relaxation parameter $\Lambda$, which nullifies $\overline{\delta v}$ for $\Lambda=3 / 8$ in this horizontal channel setup. When degrading the TRT to the singlerelaxation-time (SRT) collision model [50], i.e. setting $\tau^{+}=\tau^{-}=\tau$, then the relaxation footprint becomes $\Lambda \propto v^{2}$, which leads to a nonlinear dependence on the model viscosity. This succinctly explains the inconsistency of SRT schemes to model Brinkman flows [13]; a deeper analysis on this subject is given by $[14,32,33]$.

The velocity profile, subject to boundary conditions $u(y=$ $\pm h)=0$ and $v(y= \pm h)=v_{0}=$ const, obeys the trivial solution $v=v_{0}$ along the $v$ component, whereas the $u$ component, in nondimensional form $\bar{u}=u v /\left(g_{x} h^{2}\right)$, has the solution:

$$
\begin{aligned}
\bar{u}= & \operatorname{Da} \quad\left[1+\exp (\bar{y} \psi)\left(\frac{\sinh (\psi)}{\sinh (\psi \zeta)} \sinh (\bar{y} \psi \zeta)\right.\right. \\
& \left.\left.-\frac{\cosh (\psi)}{\cosh (\psi \zeta)} \cosh (\bar{y} \psi \zeta)\right)\right],
\end{aligned}
$$

with $\bar{y}=y / h \in[-1,1]$.

The following parameters in Eq. (14) are defined: $\psi=$ $\left(\frac{\operatorname{Re}}{2}\right) \frac{(1+\gamma)}{(1+\overline{\delta v})}, \quad \zeta=\left(\frac{4(1+\overline{\delta v})}{(1+\gamma)^{2} \operatorname{Re}^{2} \mathrm{Da}}+1\right)^{1 / 2}, \quad$ with the physical nondimensional groups $\mathrm{Da}=\frac{k}{h^{2}}$ (Darcy number) and $\operatorname{Re}=\frac{h v_{0}}{v}$ 

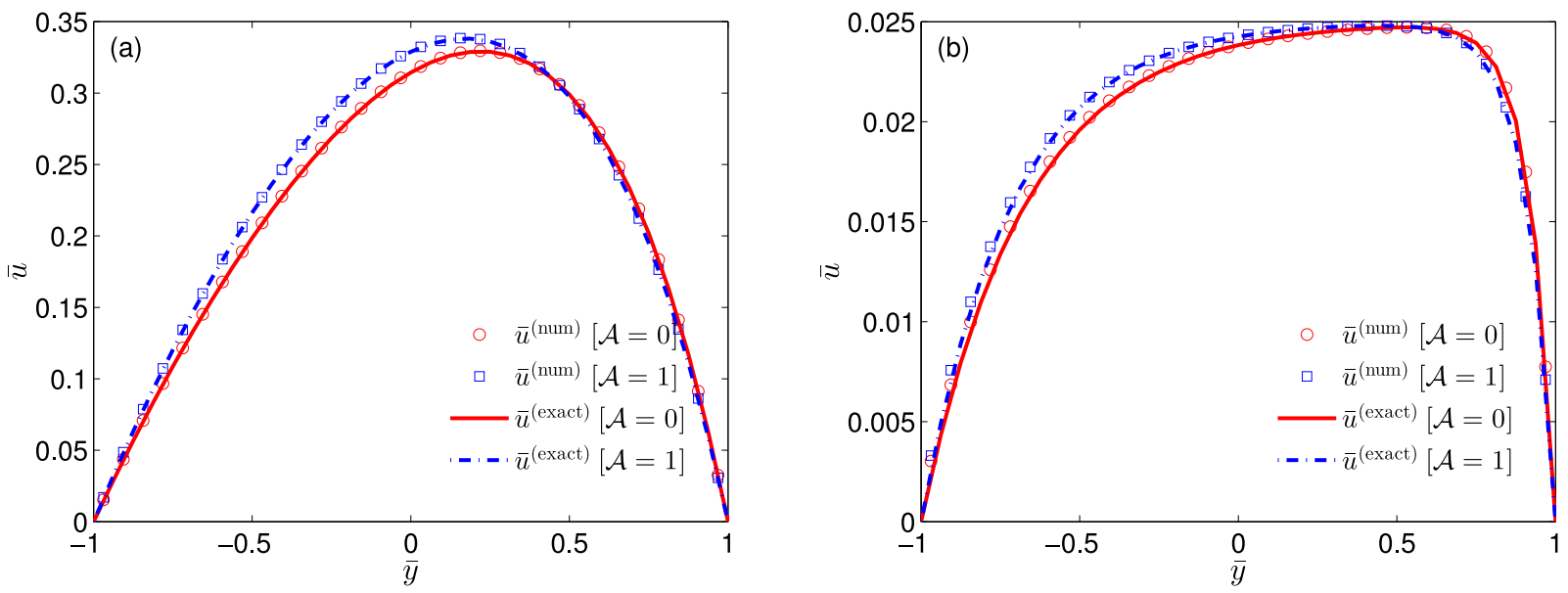

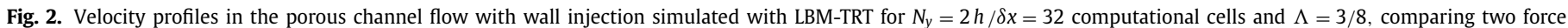

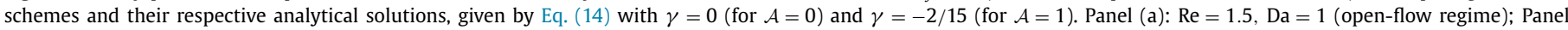
(b): $\operatorname{Re}=10, \mathrm{Da}=0.025$ (impermeable regime).

(Reynolds numbers). The physical solution for this problem should feature $\gamma=0$ (set by $\mathcal{A}=0$ ), whereas the case $\gamma \neq 0$ (set by $\mathcal{A}=1$ ) establishes a different physical problem. Fig. 2 illustrates this difference showing that for the same Da and Re two clearly distinct $u$ velocity profiles are recovered, each agreeing with the respective theoretical predictions. ${ }^{2}$

We note that the numerical artefact $\gamma$, defined in Eq. (12), can be re-written in terms of non-dimensional groups as $\gamma=$ $-\mathcal{A} \frac{6 \mathrm{Ma}^{2}}{\mathrm{Re}^{2} \mathrm{Da}}$, with $\mathrm{Ma}=\frac{v_{0}}{c}$ the Mach number. The $\gamma$ impact is therefore twofold, depending on how we handle Ma in LBM simulations.

Under the convective scaling we fix $\mathrm{Ma}=\mathcal{O}(1)$ [40], hence $\gamma$ also becomes fixed. Fig. 3 demonstrates the mesh convergence results in this case. The second-order mesh convergence of LBM towards Eq. (14) is only established providing the correct $\mathcal{A}$ value is set in the problem solution. For $\mathcal{A}=0$ the LBM numerical solution converges as a second-order scheme, as expected, towards the physical solution, in which $\gamma=0$. For $\mathcal{A}=1$ the LBM is also second-order convergent, but towards a different unphysical physical problem, given by Eq. (14) with $\gamma \neq 0$ fixed. At the same time, the LBM with $\mathcal{A}=1$, but used to solve the physical problem instead, as given by Eq. (14) with $\gamma=0$, then becomes zeroth-order convergent. That is, the scaling that formally justifies the forcing scheme with $\mathcal{A}=1$ for time-dependent problems, leads in turn to a non-convergent behavior towards the physical solution at steadystate.

Under the diffusive scaling, the Mach number is Ma $=\mathcal{O}(\delta x)$ [40]. Therefore, with fixed Re and Da, the numerical artefact $\gamma$ formally scales as $\mathcal{O}\left(\delta x^{2}\right)$; recall, $\gamma \propto \mathrm{Ma}^{2}$. In this case, the importance of the $\gamma$ error term may be overlooked as being the same order of the LBM discretization. However, the structure of this error term is different. Contrary to the typical LBM-TRT spatial discretization errors, the $\gamma$ artefact introduces a non-linear dependence on viscosity, which contaminates the overall viscosity-independent structure of the TRT numerical errors. This general shortcoming - caused by LBM force schemes with a non-zero second order velocity moment, particularized here for the $\mathcal{A}=1$ case - is studied in more details in [52]. Fig. 4 quantifies the impact of such a $v$-dependence on the $\mathrm{L}_{2}(u)$ accuracy of LBM-TRT solutions under the diffusive scaling. Comparing panels (a) and (b) in Fig. 4 it is confirmed that, in

\footnotetext{
${ }^{2}$ For clarity, the present analysis takes $\Lambda=3 / 8$, which sets $\overline{\delta v}=0$, thus making $\gamma$ the only effective force-related error throughout. The impact of $\overline{\delta v}$ in the Brinkman model simulation of porous media flows has been studied in details in $[13,14,32,33]$.
}

accordance to $\gamma$ in Eq. (12), the effect of $v$ increases the smaller is $k$ (with $\delta x$ fixed), and scales as $v^{2}$ for any fixed $k$. Fig. 5 confirms this defect from a different perspective; it shows the effect of $v$ on the quality of the velocity profiles, again for fixed Re and Da. The marked transfigurations in the shape of the profiles, caused by varying $v$, are unphysical and particularly alarming when simulating the more impermeable regime, Da $\ll 1$, which is a scenario often found in literature, e.g., [12,15,32]. These results report the gains in using a force scheme with $\mathcal{A}=0$, rather than $\mathcal{A}=1$, for the simulation of steady incompressible porous flow problems. The possible gains by switching to $\mathcal{A}=1$ when solving the timedependent regime of this problem class have not been quantified, to the best of our knowledge; to fill this gap we envisage, for example, the benchmark provided in Section 3 of [35] as an interesting route for the time-dependent analysis.

\subsection{Rotating channel Poiseuille flow}

Consider a constant acceleration field $g_{x}$ that drives an incompressible fluid inside a channel that undergoes rotation about an axis perpendicular to its own axis. The channel angular speed is $\vec{\Omega}=\Omega_{z} \vec{i}_{z}$, where $\Omega_{z}=$ const $>0$, see Fig. 1 (b). Under these conditions the acceleration field is given by:

$a_{x}=g_{x}, \quad a_{y}=-2 \Omega_{z} u$.

Note, in principle, the $a_{x}$ component should also feature the Coriolis acceleration term $2 \Omega_{z} v$. It is omitted though as the $v$ component is identically zero in this problem, due to the impermeability boundary condition $v(y= \pm h)=0$.

With the $v=0$ solution established and the introduction of Eq. (15) into Eq. (8), then the LBM describes this problem at discrete level exactly as follows:

$$
g_{x}+v \frac{u_{j+1}-2 u_{j}+u_{j-1}}{\delta x^{2}}+\mathcal{A} \frac{6 \Omega_{z} v}{c^{2}}\left(\frac{u_{j+1}^{2}-u_{j-1}^{2}}{2 \delta x}\right)=0 .
$$

Eq. (16) reproduces the following equivalent differential equation:

$g_{x}+v \partial_{y y} u+\mathcal{A} \frac{6 \Omega_{z} v}{c^{2}} \partial_{y} u^{2}=0$.

Eq. (17) subject to $u(y= \pm h)=0$ is not accessible to a closedform analytical solution. Hence, we follow the ideas of [17] and tackle this non-linear problem through a perturbation procedure. 

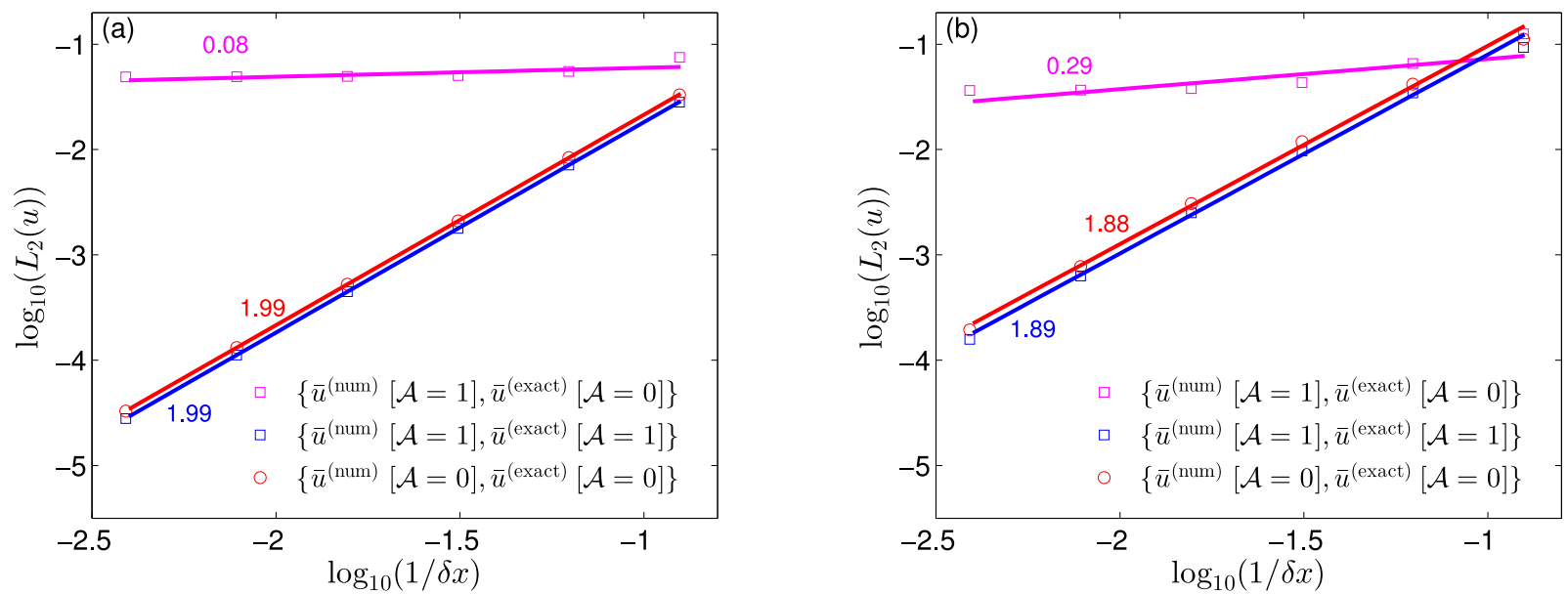

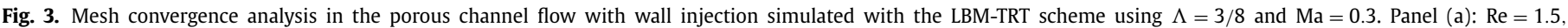
$\mathrm{Da}=1$ (open-flow regime); Panel (b): $\mathrm{Re}=10, \mathrm{Da}=0.025$ (impermeable regime). Mesh convergence rates are quantified over lines.
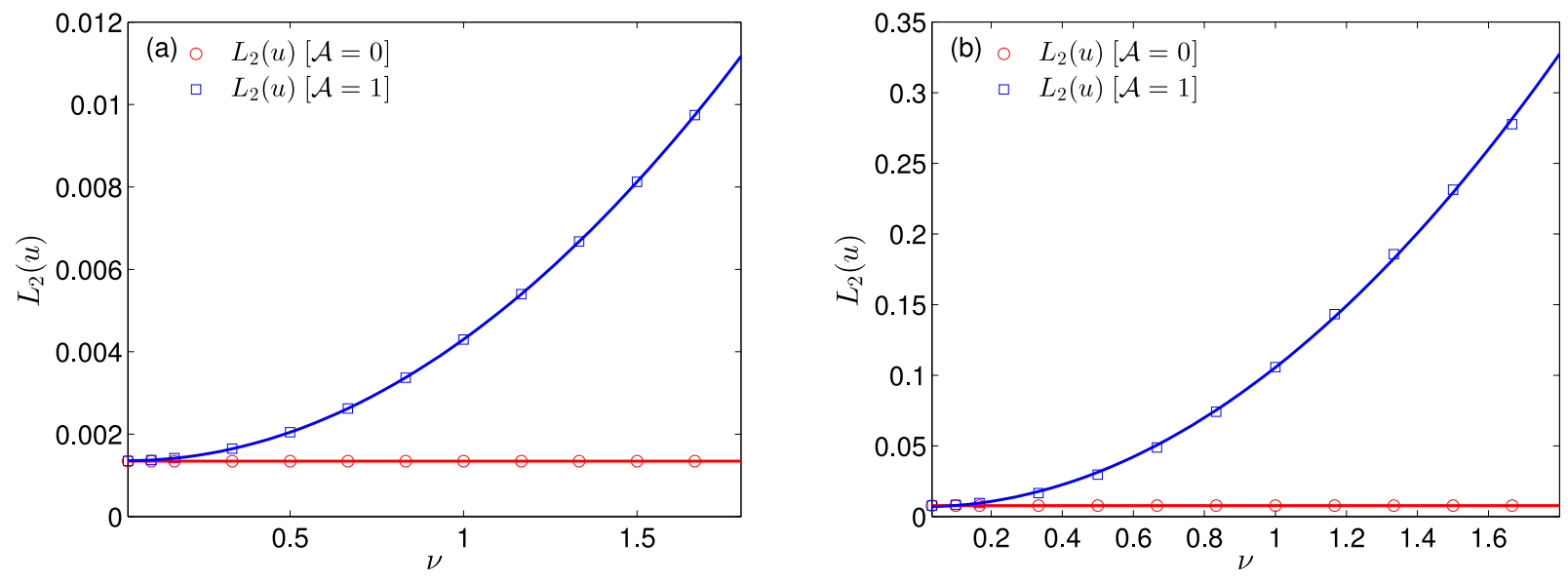

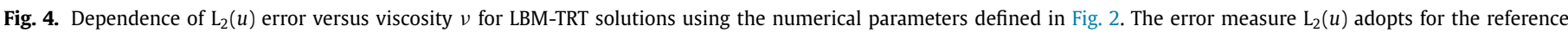
solution $u^{\text {(exact) }}$ the physical solution given by Eq. (14) with $\gamma=0$. Panel (a): $\operatorname{Re}=1.5$, Da $=1$ (open-flow regime); Panel (b): Re $=10$, Da $=0.025$ (impermeable regime).
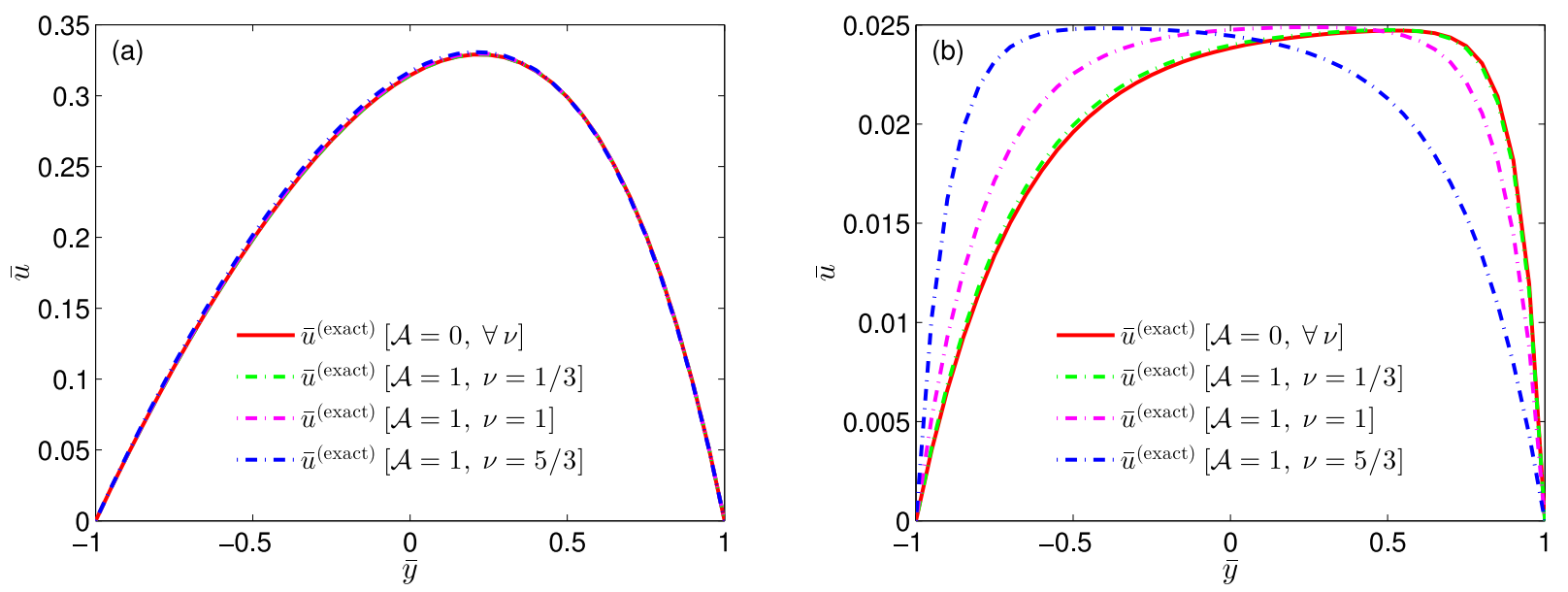

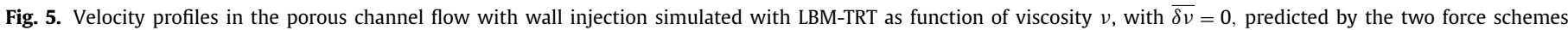
$\mathcal{A}=0$ and $\mathcal{A}=1$. Panel (a): $\operatorname{Re}=1.5, \mathrm{Da}=1$ (open-flow regime); Panel (b): $\operatorname{Re}=10, \mathrm{Da}=0.025$ (impermeable regime).

For that, we express $u$ in non-dimensional form $\bar{u}=u v /\left(g_{x} h^{2}\right)$ and assume the $\bar{u}$ velocity in Eq. (17) can be represented by $\bar{u}=$ $\bar{u}^{(0)}+\epsilon \bar{u}^{(1)}+\epsilon^{2} \bar{u}^{(2)}+\epsilon^{3} \bar{u}^{(3)}+\mathcal{O}\left(\epsilon^{4}\right)$, with the smallness parameter defined as:

$\epsilon=\mathcal{A} \frac{6 \Omega_{z} g_{x} h^{3}}{c^{2} v}$
After some lengthy algebra, the asymptotic solution of Eq. (17), represented in terms of $\bar{u}$ up to $\mathcal{O}\left(\epsilon^{4}\right)$, is given by:

$$
\begin{aligned}
\bar{u}(\bar{y})= & \frac{1}{2}\left(1-\bar{y}^{2}\right)\left(1+\frac{\epsilon}{30} \bar{y}\left(3 \bar{y}^{2}-7\right)+\frac{\epsilon^{2}}{1440}\left(1-\bar{y}^{2}\right)^{2}\left(9 \bar{y}^{2}-25\right)\right. \\
& \left.+\frac{\epsilon^{3}}{2494800} \bar{y}\left(3969 \bar{y}^{8}-28756 \bar{y}^{6}+75590 \bar{y}^{4}-92116 \bar{y}^{2}+56417\right)+O\left(\epsilon^{4}\right)\right),
\end{aligned}
$$



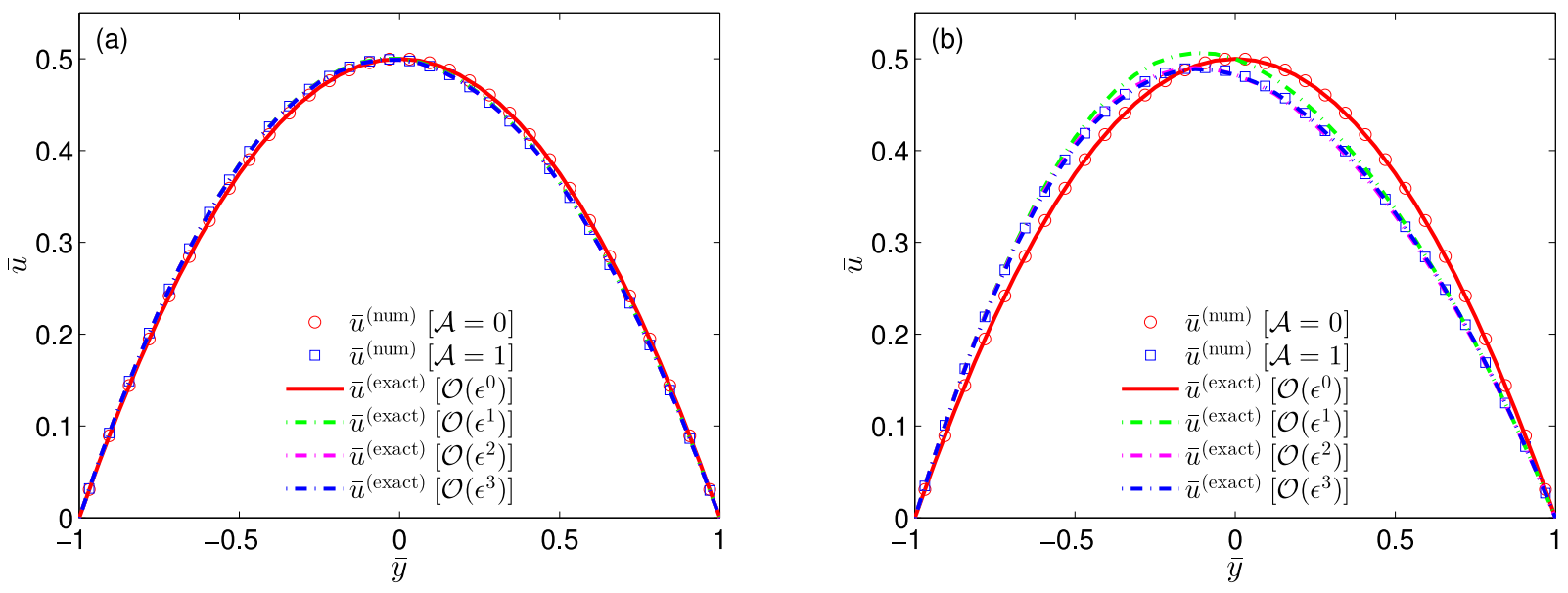

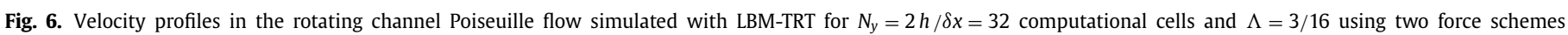
(with $\mathcal{A}=0$ and $\mathcal{A}=1$ ) versus the analytical solutions given by Eq. (19) truncated at different $\mathcal{O}\left(\epsilon^{n}\right)$-orders, with $n=\{0,1,2,3\}$. Panel (a): $\epsilon=0.25 ;$ Panel (b): $\epsilon=1$.
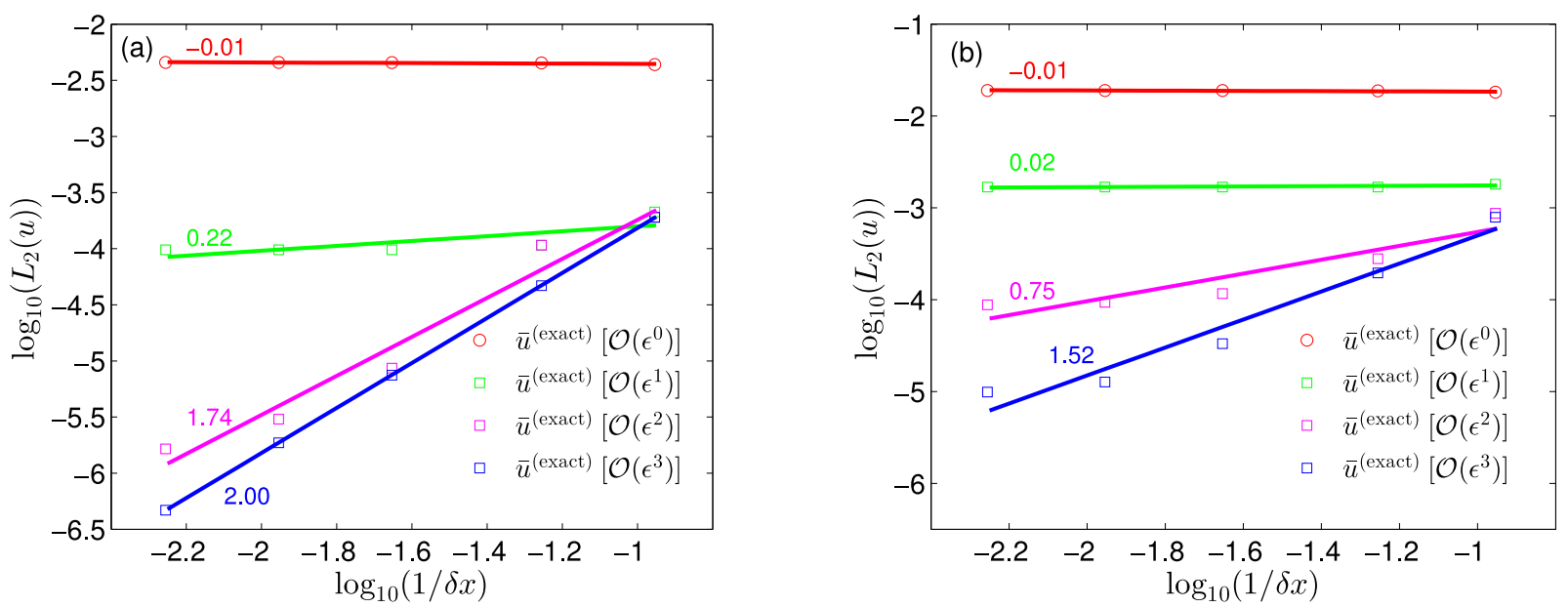

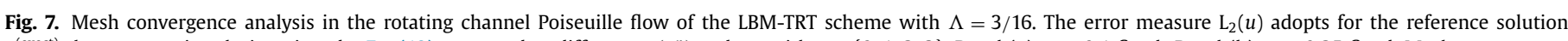

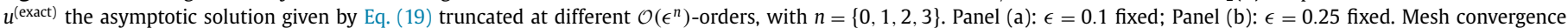
rates are quantified over lines.

with $\bar{y}=y / h \in[-1,1]$.

Note, the physical problem of the rotating channel Poiseuille flow is established for $\epsilon=0$, which recovers the parabolic profile in Eq. (19), i.e. $\bar{u}(\bar{y})=\frac{1}{2}\left(1-\bar{y}^{2}\right)$. This solution coincides with the outcome of LBM with $\mathcal{A}=0$, i.e. with the first-order force model. ${ }^{33}$ In contrast, the LBM with $\mathcal{A}=1$, i.e. with the second order force expansion, establishes a different physical problem as given by Eq. (19) with $\epsilon \neq 0$. Fig. 6 illustrates the $u$ velocity profiles recovered by LBM with $\mathcal{A}=0$ or $\mathcal{A}=1$, and compares them to the theoretical profiles, predicted by different $\mathcal{O}(\epsilon)$ asymptotic solutions in Eq. (19). The different matchings are coherent with the analytical predictions.

Again, the numerical artefact $\epsilon$, defined in Eq. (18), can be re-written in terms of non-dimensional groups as follows: $\epsilon=$ $\mathcal{A} \frac{6 \mathrm{Ma}^{2}}{\mathrm{EkRe}}$, where $\mathrm{Ek}=\frac{v}{\Omega_{z} h^{2}}$ (Ekman number), $\mathrm{Re}=\frac{u_{0} h}{v}$ (Reynolds

\footnotetext{
${ }^{3}$ The recovering of the parabolic solution is a bulk result. Hence, it holds independently of the boundary scheme employed. If the bounce-back (BB) rule is used, as considered herein, then the exact location of the no-slip zero velocity state depends on the relaxation parameters. In this particular setup, considering a halfway wall [7,27], the exact solution for $\mathcal{A}=0$ is recovered with $\Lambda=3 / 16$ in TRT (or $v=c^{2} \delta t / \sqrt{48}$ in SRT).
}

number) and $\mathrm{Ma}=\frac{u_{0}}{c}$ (Mach number), with velocity reference scale as $u_{0}=\frac{g_{x} h^{2}}{v}$. This permits understanding the impact of $\epsilon$, based on the Ma setting.

Under the convective scaling we fix $\mathrm{Ma}=\mathcal{O}(1)$ [40]. As a result, for fixed Ek and Re, the LBM with $\mathcal{A}=1$ becomes a secondorder numerical scheme for a problem distinct from the physical one. Fig. 7 demonstrates the mesh convergence analysis taking different $\mathcal{O}(\epsilon)$ approximations of the predicted theoretical solution, as given by Eq. (19). The plots confirm that the outcomes of LBM with $\mathcal{A}=1$ tend to a second-order convergence towards Eq. (17) as $\mathcal{O}(\epsilon)$ is increased, which confirms that LBM with $\mathcal{A}=1$ is indeed reproducing the differential system given by Eq. (17). The parabolic solution, as physically expected to be recovered in this problem, is only retrieved for LBM with $\mathcal{A}=0$, whereas the second-order force scheme $\mathcal{A}=1$ leads to a zeroth-order accurate representation of the (physical) $\mathcal{O}\left(\epsilon^{0}\right)$ solution.

Under the diffusive scaling, the Mach number is $\mathrm{Ma}=\mathcal{O}(\delta x)$ [40]. Therefore, for Ek and Re fixed, the numerical artefact represented by $\epsilon$ will formally scale as $\mathcal{O}\left(\delta x^{2}\right)$; recall, $\epsilon \propto \mathrm{Ma}^{2}$. Although this case may appear favourable, since the $\epsilon$ error decreases with the square of mesh resolution, with this scaling we tolerate $\epsilon$ to act as an unphysical viscosity-dependent numerical error. Fig. 8 

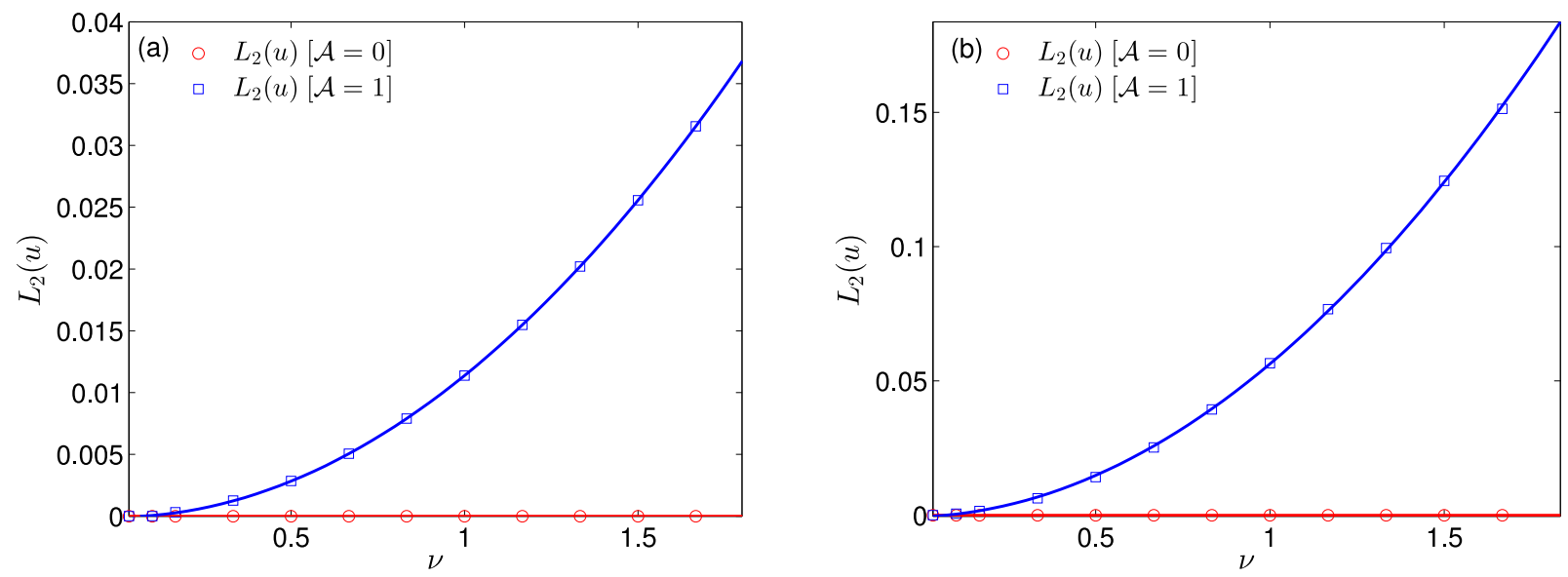

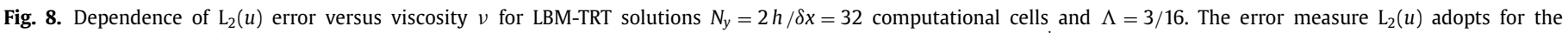

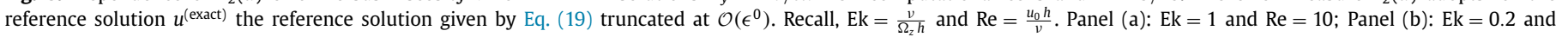
$\operatorname{Re}=10$.
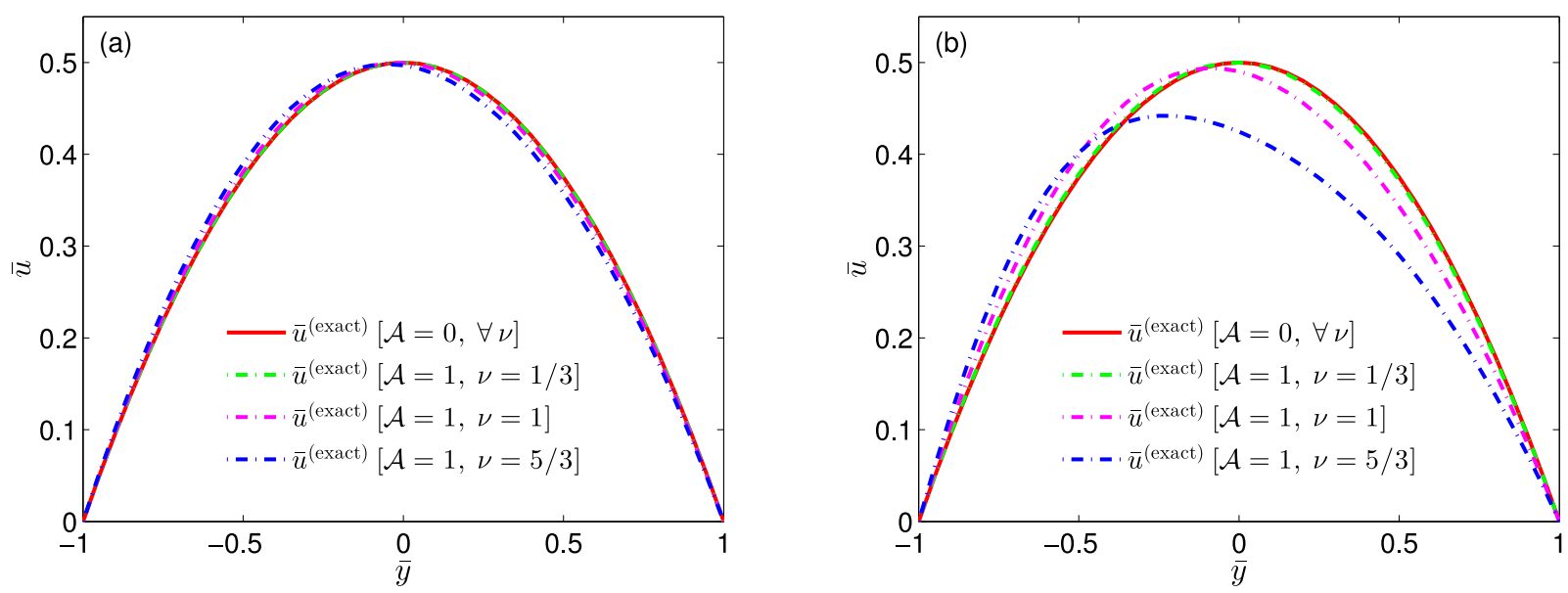

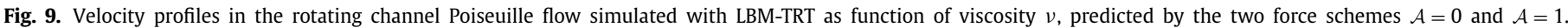
Recall, Ek $=\frac{v}{\Omega_{z} h}$ and $\operatorname{Re}=\frac{u_{0} h}{v}$. Panel (a): Ek $=1$ and $\operatorname{Re}=10$; Panel (b): Ek $=0.2$ and $\operatorname{Re}=10$.

quantifies the impact of the $v$-dependence on the $\mathrm{L}_{2}(u)$ accuracy of the TRT steady solutions obtained under the diffusive scaling. Fig. 9 illustrates the negative impact of this viscosity effect on the quality of the velocity profiles, which for Re and Ek fixed are severally distorted with the $v$ values. These results clearly establish how unreliable LBM with $\mathcal{A}=1$ is in the modeling of the Coriolis acceleration term $[18,19]$ for steady incompressible flows, a choice that is particularly harmful in the simulation of the $\mathrm{Ek} \ll 1$ regime where the $\epsilon$ force error tends to become dominant, see panel (b) in Fig. 9. On the other hand, the benefits of using a force model with $\mathcal{A}=1$ have been reported in [17] for the modeling of rotating compressible flows. The case of time-dependent incompressible flows, although theoretically conceivable to reach a superior accuracy with the force scheme using $\mathcal{A}=1$, a numerical proof of the advantage of $\mathcal{A}=1$ over $\mathcal{A}=0$ is still lacking, up to the best of or knowledge.

Finally, we comment on the effect of the intrinsic force discretization error (i.e. Error (I) in Eq. (9)), which is absent from Eq. (16) because the Laplacian of $a_{x}$ vanishes for the momentum balance along the $\mathrm{x}$-direction. Yet, this term still impacts this problem, but for the momentum balance along the $y$-direction. Looking at the solution of the NSE satisfied by this problem along the $y$ direction we arrive at the hydrostatic momentum balance: $-\partial_{y} P+$ $\bar{\rho} a_{y}=0$, with $a_{y}=-2 \Omega_{z} u$, see Eq. (15). It turns out that the same problem when tackled by LBM (derivations can be found in $[10,11,32])$ satisfies the discrete momentum balance $-\frac{1}{\bar{\rho}} \frac{P_{j+1}-P_{j-1}}{2 \delta x}+$ $\left(a_{y}\right)_{j}+\frac{\delta x^{2}}{4} \frac{\left(a_{y}\right)_{j+1}-2\left(a_{y}\right)_{j}+\left(a_{y}\right)_{j-1}}{\delta x^{2}}=0$, which when translated into this case reads $-\frac{1}{\bar{\rho}} \frac{\delta P_{j+1}^{2}-P_{j-1}}{2 \delta x}-2 \Omega_{z} u_{j}-\frac{\delta x^{2}}{2} \Omega_{z} \frac{u_{j+1}-2 u_{j}+u_{j-1}}{\delta x^{2}}=0$ or equivalently $-\frac{1}{\bar{\rho}} \frac{P_{j+1}-P_{j-1}}{2 \delta x}-2 \Omega_{z} \frac{u_{j+1}+2 u_{j}+u_{j-1}}{4}=0$, where the term $\frac{u_{j+1}+2 u_{j}+u_{j-1}}{4}$ represents the non-local stencil integration of the spatially-varying contribution in the body force. The reason why the discrete Laplacian of $a_{y}$ in the $y$-momentum is independent of the relaxation rates, contrasting with the $\Lambda$-dependence on the discrete Laplacian of $a_{x}$ in Eq. (8), is explained by the fact that the discrete momentum balance established here represents a static balance, i.e. a system at quasi-equilibrium where all relaxation contributions vanish; this is intuitively expected as relaxation contributions come associated with non-equilibrium phenomena.

\section{Conclusions}

By analytically solving the LBM-TRT scheme for a specific steady-state force-driven flow configuration, we exactly established its discrete momentum balance. This study permitted identifying the existence of two spatially-dependent force error sources. 
The first one is due to the non-local spatial discretization of the forcing term, given by the discrete Laplacian of the body force. This is a defect inherent to LBM. Such a term is responsible for corrupting the discrete momentum balance with a non-linear viscosity dependent term in the single-relaxation-time schemes that, however, can be avoided with the TRT scheme, through its free-tunable relaxation degree of freedom $\Lambda$ as previously indicated in [14,32,33].

The other error source arises from the use of the second-order velocity space expansion in the force formulation. The introduced second-order velocity moment interferes with the discrete momentum balance, via a spurious first-order derivative, whose negative impact is shown to produce the following defects. With LBM simulations running under the convective scaling this second-order term renders the forced LBM a second-order spatial convergent scheme towards a differential system different from the physical one or it makes LBM a zeroth-order convergent scheme towards the physical problem, i.e. LBM becomes an inconsistent numerical scheme. Note, this convective scaling analysis is important not only because of its interest in specific flow simulations, but essentially because only this scaling permits theoretically justifying the second-order expansion of the forcing term in timedependent problems. When simulations run under the diffusive scaling the aforementioned second-order moment term is allowed to change with the grid resolution, but at costs of contaminating the overall structure of the TRT numerical errors, rendering the (non-dimensional) forced TRT steady-state solutions unavoidably viscosity-dependent.

Opposingly, the first-order force expansion operated in the LBM-TRT framework consistently reproduces spatially-varying body forces, reaching the same solution under convective or diffusive scalings, as physically expected at steady-state. These conclusions are in agreement with $[38,39]$, and support the first-order force scheme in steady hydrodynamics.

Given that the modeling of force-driven unsteady flows seems, however, more accurate with a second-order force scheme, e.g. $[30,39]$, we pinpoint the possibility of switching the structure of the source term on the fly, i.e. automatically during the LBM simulation. A straightforward idea for this realization consists in flagging the $\mathcal{A}$ value within the force scheme, Eq. (3), in terms of the time evolution of some relevant macroscopic quantity, e.g. the flow velocity. As by default, the LBM evolves as a time-marching procedure, the algorithm can be initialized with $\mathcal{A}=1$ and so maintained until the simulation is about to reach the steadystate criterion; at this point, we switch to $\mathcal{A}=0$ so that, right after, when the steady-state regime is reached the corresponding time-independent solution supports the consistency properties discussed in this work. This procedure mimics the protocol employed in the flagging of the anti-numerical-diffusion correction popularized in the simulation of time-varying advection-diffusion problems $[43,45]$.

\section{Declaration of Competing Interest}

The author declares that he has no known competing financial interests or personal relationships that could have appeared to influence the work reported in this paper.

\section{CRediT authorship contribution statement}

Goncalo Silva: Writing - original draft.

\section{Acknowledgments}

It is acknowledged the financial support from Fundação para a Ciência e Tecnologia (FCT) through Grant No.
SFRH/BPD/111228/2015, and FCT, through IDMEC, under LAETA, project UID/EMS/50022/2019.

\section{Appendix A. Analytical solution of LBM-TRT based on procedure of He et al. [46]}

The starting point in He et al. [46] derivation begins with the first order velocity moment:

$\sum_{q=1}^{Q-1} c_{q x} f_{q}(y)=c\left(f_{1}^{j}-f_{3}^{j}+f_{5}^{j}-f_{6}^{j}-f_{7}^{j}+f_{8}^{j}\right)=\bar{\rho} u_{j}-\frac{\delta t}{2} \bar{\rho}\left(a_{x}\right)_{j}$

The explicit content of each population in Eq. (A.1) is then determined. The first step expands Eq. (6) for the two main links $q=1$ and 3 , as follows:

$$
\begin{aligned}
f_{1}^{j}= & \left(\frac{\tau^{+}-\tau^{-}}{\tau^{+}+\tau^{-}}\right) f_{3}^{j}+\frac{1}{3}\left(\frac{2 \tau^{-}}{\tau^{+}+\tau^{-}}\right) \frac{P_{j}}{c^{2}}+\frac{1}{3}\left(\frac{2 \tau^{+}}{\tau^{+}+\tau^{-}}\right) \bar{\rho}\left(\frac{u_{j}}{c}\right) \\
& +\frac{1}{3}\left(\frac{2 \tau^{-}}{\tau^{+}+\tau^{-}}\right) \bar{\rho}\left(\frac{u_{j}^{2}}{c^{2}}-\frac{1}{2} \frac{v_{j}^{2}}{c^{2}}\right) \\
& +\frac{1}{3} \tau^{+}\left(\frac{2 \tau^{-}-1}{\tau^{+}+\tau^{-}}\right) \delta t \bar{\rho}\left(\frac{\left(a_{x}\right)_{j}}{c}\right) \\
& +\mathcal{A} \frac{1}{3} \tau^{-}\left(\frac{2 \tau^{+}-1}{\tau^{+}+\tau^{-}}\right) \delta t \bar{\rho}\left(2 \frac{u_{j}\left(a_{x}\right)_{j}}{c^{2}}-\frac{v_{j}\left(a_{y}\right)_{j}}{c^{2}}\right), \\
f_{3}^{j}= & \left(\frac{\tau^{+}-\tau^{-}}{\tau^{+}+\tau^{-}}\right) f_{1}^{j}+\frac{1}{3}\left(\frac{2 \tau^{-}}{\tau^{+}+\tau^{-}}\right) \frac{P_{j}}{c^{2}} \\
& +\frac{1}{3}\left(\frac{2 \tau^{+}}{\tau^{+}+\tau^{-}}\right) \bar{\rho}\left(-\frac{u_{j}}{c}\right)+\frac{1}{3}\left(\frac{2 \tau^{-}}{\tau^{+}+\tau^{-}}\right) \bar{\rho}\left(\frac{u_{j}^{2}}{c^{2}}-\frac{1}{2} \frac{v_{j}^{2}}{c^{2}}\right) \\
& +\frac{1}{3} \tau^{+}\left(\frac{2 \tau^{-}-1}{\tau^{+}+\tau^{-}}\right) \delta t \bar{\rho}\left(-\frac{\left(a_{x}\right)_{j}}{c}\right) \\
& +\mathcal{A} \frac{1}{3} \tau^{-}\left(\frac{2 \tau^{+}-1}{\tau^{+}+\tau^{-}}\right) \delta t \bar{\rho}\left(2 \frac{u_{j}\left(a_{x}\right)_{j}}{c^{2}}-\frac{v_{j}\left(a_{y}\right)_{j}}{c^{2}}\right) .
\end{aligned}
$$

The second step expands Eq. (6) for the diagonal links, from $q=5$ to 8 , as follows:

$$
\begin{aligned}
f_{5}^{j}= & \left(\frac{1}{2 \tau^{-}}-\frac{1}{2 \tau^{+}}\right) f_{7}^{j-1}+\left(1-\frac{1}{2 \tau^{-}}-\frac{1}{2 \tau^{+}}\right) f_{5}^{j-1} \\
& +\frac{1}{12 \tau^{+}} \frac{P_{j-1}}{c^{2}}+\frac{1}{12 \tau^{-}} \bar{\rho}\left(\frac{u_{j-1}}{c}+\frac{v_{j-1}}{c}\right) \\
& +\frac{1}{12 \tau^{+}} \bar{\rho}\left(\frac{u_{j-1}^{2}}{c^{2}}+\frac{v_{j-1}^{2}}{c^{2}}+3 \frac{u_{j-1} v_{j-1}}{c^{2}}\right) \\
& +\frac{1}{12}\left(1-\frac{1}{2 \tau^{-}}\right) \delta t \bar{\rho}\left(\frac{\left(a_{x}\right)_{j-1}}{c}+\frac{\left(a_{y}\right)_{j-1}}{c}\right) \\
& +\mathcal{A} \frac{1}{12}\left(1-\frac{1}{2 \tau^{+}}\right) \delta t \bar{\rho}\left(2 \frac{u_{j-1}\left(a_{x}\right)_{j-1}}{c^{2}}+2 \frac{v_{j-1}\left(a_{y}\right)_{j-1}}{c^{2}}\right. \\
& \left.+3 \frac{u_{j-1}\left(a_{y}\right)_{j-1}}{c^{2}}+3 \frac{v_{j-1}\left(a_{x}\right)_{j-1}}{c^{2}}\right),
\end{aligned}
$$

$$
\begin{aligned}
f_{6}^{j}= & \left(\frac{1}{2 \tau^{-}}-\frac{1}{2 \tau^{+}}\right) f_{8}^{j-1}+\left(1-\frac{1}{2 \tau^{-}}-\frac{1}{2 \tau^{+}}\right) f_{6}^{j-1} \\
& +\frac{1}{12 \tau^{+}} \frac{P_{j-1}}{c^{2}}+\frac{1}{12 \tau^{-}} \bar{\rho}\left(-\frac{u_{j-1}}{c}+\frac{v_{j-1}}{c}\right) \\
& +\frac{1}{12 \tau^{+}} \bar{\rho}\left(\frac{u_{j-1}^{2}}{c^{2}}+\frac{v_{j-1}^{2}}{c^{2}}-3 \frac{u_{j-1} v_{j-1}}{c^{2}}\right) \\
& +\frac{1}{12}\left(1-\frac{1}{2 \tau^{-}}\right) \delta t \bar{\rho}\left(\frac{\left(a_{x}\right)_{j-1}}{c}+\frac{\left(a_{y}\right)_{j-1}}{c}\right)
\end{aligned}
$$




$$
\begin{aligned}
& +\mathcal{A} \frac{1}{12}\left(1-\frac{1}{2 \tau^{+}}\right) \delta t \bar{\rho}\left(2 \frac{u_{j-1}\left(a_{x}\right)_{j-1}}{c^{2}}+2 \frac{v_{j-1}\left(a_{y}\right)_{j-1}}{c^{2}}\right. \\
& \left.-3 \frac{u_{j-1}\left(a_{y}\right)_{j-1}}{c^{2}}-3 \frac{v_{j-1}\left(a_{x}\right)_{j-1}}{c^{2}}\right),
\end{aligned}
$$

$$
\begin{aligned}
f_{7}^{j}= & \left(\frac{1}{2 \tau^{-}}-\frac{1}{2 \tau^{+}}\right) f_{5}^{j+1}+\left(1-\frac{1}{2 \tau^{-}}-\frac{1}{2 \tau^{+}}\right) f_{7}^{j+1} \\
& +\frac{1}{12 \tau^{+}} \frac{P_{j+1}}{c^{2}}+\frac{1}{12 \tau^{-}} \bar{\rho}\left(-\frac{u_{j+1}}{c}-\frac{v_{j+1}}{c}\right) \\
& +\frac{1}{12 \tau^{+}} \bar{\rho}\left(\frac{u_{j+1}^{2}}{c^{2}}+\frac{v_{j+1}^{2}}{c^{2}}+3 \frac{u_{j+1} v_{j+1}}{c^{2}}\right) \\
& +\frac{1}{12}\left(1-\frac{1}{2 \tau^{-}}\right) \delta t \bar{\rho}\left(-\frac{\left(a_{x}\right)_{j+1}}{c}-\frac{\left(a_{y}\right)_{j+1}}{c}\right) \\
& +\mathcal{A} \frac{1}{12}\left(1-\frac{1}{2 \tau^{+}}\right) \delta t \bar{\rho}\left(2 \frac{u_{j+1}\left(a_{x}\right)_{j+1}}{c^{2}}+2 \frac{v_{j+1}\left(a_{y}\right)_{j+1}}{c^{2}}\right. \\
& \left.+3 \frac{u_{j+1}\left(a_{y}\right)_{j+1}}{c^{2}}+3 \frac{v_{j+1}\left(a_{x}\right)_{j+1}}{c^{2}}\right),
\end{aligned}
$$

$$
-\left(\frac{1}{2 \tau^{-}}-\frac{1}{2 \tau^{+}}\right)\left(f_{5}^{j+1}-f_{6}^{j+1}\right) .
$$

Introduction of the three results, Eqs. (A.8), (A.9) and (A.10), into the first-order velocity moment, Eq. (A.1), yields:

$$
\begin{aligned}
\frac{1}{c} \bar{\rho} u_{j}-\frac{\delta t}{2 c} \bar{\rho}\left(a_{x}\right)_{j} \\
=\frac{1}{6 \tau^{-} c} \bar{\rho}\left(4 \tau^{-} u_{j}+u_{j-1}+u_{j+1}\right) \\
\quad+\frac{1}{2 \tau^{+} c^{2}} \bar{\rho}\left(u_{j-1} v_{j-1}-u_{j+1} v_{j+1}\right) \\
\quad+\left(1-\frac{1}{2 \tau^{-}}\right) \frac{\delta t}{c} \bar{\rho}\left(4 \tau^{-}\left(a_{x}\right)_{j}+\left(a_{x}\right)_{j-1}+\left(a_{x}\right)_{j+1}\right) \\
\quad+\mathcal{A}\left(1-\frac{1}{2 \tau^{+}}\right) \frac{\delta t}{2 c^{2}} \bar{\rho}\left(u_{j-1}\left(a_{y}\right)_{j-1}+v_{j-1}\left(a_{x}\right)_{j-1}\right. \\
\left.\quad-u_{j+1}\left(a_{y}\right)_{j+1}-v_{j+1}\left(a_{x}\right)_{j+1}\right) \\
\quad+\left(1-\frac{1}{2 \tau^{-}}-\frac{1}{2 \tau^{+}}\right)\left(f_{5}^{j-1}-f_{6}^{j-1}-f_{7}^{j+1}+f_{8}^{j+1}\right) \\
\quad-\left(\frac{1}{2 \tau^{-}}-\frac{1}{2 \tau^{+}}\right)\left(f_{5}^{j+1}-f_{6}^{j+1}-f_{7}^{j-1}+f_{8}^{j-1}\right) .
\end{aligned}
$$

$$
\begin{aligned}
f_{8}^{j}= & \left(\frac{1}{2 \tau^{-}}-\frac{1}{2 \tau^{+}}\right) f_{6}^{j+1}+\left(1-\frac{1}{2 \tau^{-}}-\frac{1}{2 \tau^{+}}\right) f_{8}^{j+1} \\
& +\frac{1}{12 \tau^{+}} \frac{P_{j+1}}{c^{2}}+\frac{1}{12 \tau^{-}} \bar{\rho}\left(\frac{u_{j+1}}{c}-\frac{v_{j+1}}{c}\right) \\
& +\frac{1}{12 \tau^{+}} \bar{\rho}\left(\frac{u_{j+1}^{2}}{c^{2}}+\frac{v_{j+1}^{2}}{c^{2}}-3 \frac{u_{j+1} v_{j+1}}{c^{2}}\right) \\
& +\frac{1}{12}\left(1-\frac{1}{2 \tau^{-}}\right) \delta t \bar{\rho}\left(\frac{\left(a_{x}\right)_{j+1}}{c}-\frac{\left(a_{y}\right)_{j+1}}{c}\right) \\
& +\mathcal{A} \frac{1}{12}\left(1-\frac{1}{2 \tau^{+}}\right) \delta t \bar{\rho}\left(2 \frac{u_{j+1}\left(a_{x}\right)_{j+1}}{c^{2}}+2 \frac{v_{j+1}\left(a_{y}\right)_{j+1}}{c^{2}}\right. \\
& \left.-3 \frac{u_{j+1}\left(a_{y}\right)_{j+1}}{c^{2}}-3 \frac{v_{j+1}\left(a_{x}\right)_{j+1}}{c^{2}}\right) .
\end{aligned}
$$

Subtraction of Eq. (A.2) from Eq. (A.3) gives:

$f_{1}^{j}-f_{3}^{j}=\frac{2}{3 c} \bar{\rho} u_{j}+\frac{2 \delta t}{3 c}\left(\tau^{-}-\frac{1}{2}\right) \bar{\rho}\left(a_{x}\right)_{j}$

Subtraction of Eq. (A.5) from Eq. (A.4), and of Eq. (A.6) from Eq. (A.7) gives, respectively:

$$
\begin{aligned}
f_{5}^{j}-f_{6}^{j}= & \frac{1}{2 \tau^{+}} \bar{\rho} \frac{u_{j-1} v_{j-1}}{c^{2}}+\frac{1}{6 \tau^{-}} \bar{\rho} \frac{u_{j-1}}{c} \\
& +\frac{1}{6}\left(1-\frac{1}{2 \tau^{-}}\right) \delta t \bar{\rho} \frac{\left(a_{x}\right)_{j-1}}{c} \\
& +\mathcal{A} \frac{1}{2}\left(1-\frac{1}{2 \tau^{+}}\right) \delta t \bar{\rho}\left(\frac{u_{j-1}\left(a_{y}\right)_{j-1}}{c^{2}}+\frac{v_{j-1}\left(a_{x}\right)_{j-1}}{c^{2}}\right) \\
& +\left(1-\frac{1}{2 \tau^{-}}-\frac{1}{2 \tau^{+}}\right)\left(f_{5}^{j-1}-f_{6}^{j-1}\right) \\
& +\left(\frac{1}{2 \tau^{-}}-\frac{1}{2 \tau^{+}}\right)\left(f_{7}^{j-1}-f_{8}^{j-1}\right),
\end{aligned}
$$

$$
\begin{aligned}
-f_{7}^{j}+f_{8}^{j}= & -\frac{1}{2 \tau^{+}} \bar{\rho} \frac{u_{j+1} v_{j+1}}{c^{2}}+\frac{1}{6 \tau^{-}} \bar{\rho} \frac{u_{j+1}}{c} \\
& +\frac{1}{6}\left(1-\frac{1}{2 \tau^{-}}\right) \delta t \bar{\rho} \frac{\left(a_{x}\right)_{j+1}}{c} \\
& -\mathcal{A} \frac{1}{2}\left(1-\frac{1}{2 \tau^{+}}\right) \delta t \bar{\rho}\left(\frac{u_{j+1}\left(a_{y}\right)_{j+1}}{c^{2}}+\frac{v_{j+1}\left(a_{x}\right)_{j+1}}{c^{2}}\right) \\
& -\left(1-\frac{1}{2 \tau^{-}}-\frac{1}{2 \tau^{+}}\right)\left(f_{7}^{j+1}-f_{8}^{j+1}\right)
\end{aligned}
$$

The last two terms in Eq. (A.11) can be determined with recourse to the following three relationships:

$$
\begin{aligned}
&\left(f_{5}^{j}-f_{6}^{j}-f_{7}^{j}+f_{8}^{j}\right)=\frac{1}{c} \bar{\rho} u_{j}-\frac{2 \delta t}{3 c}\left(\tau^{-}-\frac{1}{2}\right) \bar{\rho}\left(a_{x}\right)_{j}-\frac{\delta t}{2 c} \bar{\rho}\left(a_{x}\right)_{j} \\
&(\text { A.12a }) \\
&\left(f_{5}^{j+1}-f_{6}^{j+1}-f_{7}^{j-1}+f_{8}^{j-1}\right)= \frac{1}{\tau^{-}} \frac{1}{c} \bar{\rho} u_{j}-\frac{2 \delta t}{3 c}\left(1-\frac{1}{2 \tau^{-}}\right) \bar{\rho}\left(a_{x}\right)_{j} \\
&-\left(1-\frac{1}{\tau^{-}}\right)\left(f_{5}^{j}-f_{6}^{j}-f_{7}^{j}+f_{8}^{j}\right)
\end{aligned}
$$

$$
\begin{aligned}
& \left(f_{5}^{j-1}-f_{6}^{j-1}-f_{7}^{j+1}+f_{8}^{j+1}\right) \\
& =\frac{1}{c} \bar{\rho}\left(u_{j-1}+u_{j+1}\right)-\frac{2 \delta t}{3 c}\left(\tau^{-}-\frac{1}{2}\right) \bar{\rho}\left(\left(a_{x}\right)_{j-1}+\left(a_{x}\right)_{j+1}\right) \\
& \quad-\frac{\delta t}{2 c} \bar{\rho}\left(\left(a_{x}\right)_{j-1}+\left(a_{x}\right)_{j+1}\right)+\left(f_{5}^{j+1}-f_{6}^{j+1}-f_{7}^{j-1}+f_{8}^{j-1}\right)
\end{aligned}
$$

The first relationship is determined by combining Eq. (A.1) with Eq. (A.8). The second relationship results from re-writing Eq. (A.9) at $j+1$ and Eq. (A.10) at $j-1$, and then adding the two equations, with Eq. (A.8) included to simplify. The third relationship follows by writing Eq. (A.1) at $j \pm 1$ positions, and then simplifying the outcome with aid of Eq. (A.8). The ensemble of Eq. (A.12) are inserted into Eq. (A.11). After some simplifications, we arrive at the following equation:

$$
\begin{aligned}
& \frac{1}{2 c^{2}} \bar{\rho}\left(u_{j+1} v_{j+1}-u_{j-1} v_{j-1}\right) \\
& \quad-\frac{1}{3 c}\left(\tau^{+}-\frac{1}{2}\right) \bar{\rho}\left(u_{j+1}-2 u_{j}+u_{j-1}\right)-\frac{\delta t}{c} \bar{\rho}\left(a_{x}\right)_{j} \\
& +\frac{2}{3 c} \delta t \bar{\rho}\left(\left(\tau^{+}-\frac{1}{2}\right)\left(\tau^{-}-\frac{1}{2}\right)-\frac{3}{8}\right) \\
& \times\left(\left(a_{x}\right)_{j+1}-2\left(a_{x}\right)_{j}+\left(a_{x}\right)_{j-1}\right) \\
& +\mathcal{A} \frac{1}{2 c^{2}}\left(\tau^{+}-\frac{1}{2}\right) \delta t \bar{\rho}\left(u_{j+1}\left(a_{y}\right)_{j+1}+v_{j+1}\left(a_{x}\right)_{j+1}-u_{j-1}\left(a_{y}\right)_{j-1}\right. \\
& \left.-v_{j-1}\left(a_{x}\right)_{j-1}\right)=0 .
\end{aligned}
$$


Finally, multiplication of Eq. (A.13) by $c / \delta t$, and introduction of $\delta x=c \delta$ provides the equation:

$$
\begin{aligned}
& \frac{u_{j+1} v_{j+1}-u_{j-1} v_{j-1}}{2 \delta x}-\left(a_{x}\right)_{j}-\frac{1}{3} c^{2} \delta t\left(\tau^{+}-\frac{1}{2}\right) \frac{u_{j+1}-2 u_{j}+u_{j-1}}{\delta x^{2}} \\
& +\delta x^{2}\left(\frac{8 \Lambda-3}{12}\right) \frac{\left(a_{x}\right)_{j+1}-2\left(a_{x}\right)_{j}+\left(a_{x}\right)_{j-1}}{\delta x^{2}} \\
& +\mathcal{A}\left(\tau^{+}-\frac{1}{2}\right) \delta t\left(\frac{u_{j+1}\left(a_{y}\right)_{j+1}-u_{j-1}\left(a_{y}\right)_{j-1}}{2 \delta x}\right. \\
& \left.+\frac{v_{j+1}\left(a_{x}\right)_{j+1}-v_{j-1}\left(a_{x}\right)_{j-1}}{2 \delta x}\right)=0,
\end{aligned}
$$

which leads to Eq. (8).

\section{Appendix B. Analytical solution of LBM-TRT based on procedure of Ginzburg and d'Humières [14,47]}

The starting point in Ginzburg and d'Humières [14,47] derivation consists of re-formulating the LBM-TRT equations originally written in evolution form, Eq. (1), by equivalent equations in recurrence form. For this task, we adopt the compact notation of [14] defining: (i) in equilibrium $E_{q}^{ \pm}=e_{q}^{ \pm}+\Lambda^{ \pm} S_{q}^{ \pm} \delta t$ and (ii) in nonequilibrium $g_{q}^{ \pm}=-\frac{1}{\tau^{ \pm}}\left(f_{q}^{ \pm}-E_{q}^{ \pm}\right)$; so that, Eq. (1) reads as:

$f_{q}\left(\vec{x}+\vec{c}_{q} \delta t, t+\delta t\right)=\left[f_{q}+g_{q}^{+}+g_{q}^{-}\right](\vec{x}, t)$.

Based on Eq. (B.1), the steady-state recurrence conditions for the $g_{q}^{ \pm}$in LBM-TRT, originally derived in [47], are given by:

$g_{q}^{ \pm}(\vec{x})=\left[\bar{\Delta}_{q} E_{q}^{\mp}-\Lambda^{\mp} \bar{\Delta}_{q}^{2} E_{q}^{ \pm}+\left(\Lambda-\frac{1}{4}\right) \bar{\Delta}_{q}^{2} g_{q}^{ \pm}\right](\vec{x})$,

where the relaxation coefficients are defined as $\Lambda^{ \pm}=\left(\tau^{ \pm}-1 / 2\right)$ and $\Lambda=\Lambda^{+} \Lambda^{-}$, and the following first- and second-order central link-wise finite difference operators are introduced

$\bar{\Delta}_{q} \psi(\vec{x})=\frac{1}{2}\left[\psi\left(\vec{x}+\vec{c}_{q} \delta t\right)-\psi\left(\vec{x}-\vec{c}_{q} \delta t\right)\right]$,

$\bar{\Delta}_{q}^{2} \psi(\vec{x})=\psi\left(\vec{x}+\vec{c}_{q} \delta t\right)-2 \psi(\vec{x})+\psi\left(\vec{x}-\vec{c}_{q} \delta t\right)$,

where $\psi$ denotes an arbitrary quantity. The link-wise operators, Eqs. (B.3), relate to spatial centered finite difference (FD) stencils, represented along the $y$-coordinate axis, as follows:

$\bar{\Delta}_{q} \psi=\delta t c_{q y} \bar{\Delta}_{y} \psi, \quad$ with $\quad \bar{\Delta}_{y} \psi_{j}=\frac{\psi_{j+1}-\psi_{j-1}}{2 \delta x}$,

$\bar{\Delta}_{q}^{2} \psi=\delta t^{2} c_{q y}^{2} \bar{\Delta}_{y}^{2} \psi, \quad$ with $\quad \bar{\Delta}_{y}^{2} \psi_{j}=\frac{\psi_{j+1}-2 \psi_{j}+\psi_{j-1}}{\delta x^{2}}$.

Note, the node value solution is denoted as $\psi_{j}=\psi\left(y_{j}\right)$, where $y_{j}$ defines the grid node location, labelled by index $j$. In the denominator of the FD stencils $\delta x=c \delta t$ measures the vertical distance between neighboring cells.

The $x$-component of the momentum balance is determined by the first order velocity moment of Eq. (B.2), restricted to the oddorder component:

$$
\begin{aligned}
\sum_{q=1}^{Q-1} c_{q x} g_{q}^{-}= & \sum_{q=1}^{Q-1} c_{q x} \bar{\Delta}_{q} E_{q}^{+}-\Lambda^{+} \sum_{q=1}^{Q-1} c_{q x} \bar{\Delta}_{q}^{2} E_{q}^{-} \\
& +\left(\Lambda-\frac{1}{4}\right) \sum_{q=1}^{Q-1} c_{q x} \bar{\Delta}_{q}^{2} g_{q}^{-} .
\end{aligned}
$$

The momentum solvability condition is given by:

$$
\sum_{q=1}^{Q-1} \vec{c}_{q} g_{q}^{-}=\bar{\rho} \vec{a} \delta t .
$$

The proof of Eq. (B.6) results from the $g_{q}^{-}$definition, given by $g_{q}^{-}=-\frac{1}{\tau^{-}}\left(f_{q}^{-}-e_{q}^{-}-\Lambda^{-} S_{q}^{-} \delta t\right)$, and then determined as $\quad \sum_{q=1}^{Q-1} \vec{c}_{q} g_{q}^{-}=-\frac{1}{\tau^{-}} \sum_{q=1}^{Q-1} \vec{c}_{q}\left(f_{q}^{-}-e_{q}^{-}-\Lambda^{-} S_{q}^{-} \delta t\right)=$ $-\frac{1}{\tau^{-}}\left(\bar{\rho} \vec{u}-\frac{\delta t}{2} \bar{\rho} \vec{a}-\bar{\rho} \vec{u}-\Lambda^{-} \bar{\rho} \vec{a} \delta t\right)=-\frac{1}{\tau^{-}}\left(-\tau^{-} \bar{\rho} \vec{a} \delta t\right)=\bar{\rho} \vec{a} \delta t$.

Then, with the assumptions given in Section 3 it is concluded that the only non-trivial links of the LBM solution ( $g_{q}^{-}$included) are $c_{q x} c_{q y} \neq 0$. This restriction combined with Eq. (B.6) enables us to determine the exact $g_{q}^{-}$solution of this problem as [14]:

$g_{q}^{-}=3 \frac{c_{q y}^{2}}{c^{2}} S_{q}^{-} \delta t$

The introduction of Eq. (B.7) for $g_{q}^{-}$, Eq. (2) for $e_{q}^{ \pm}$and Eq. (3) for $S_{q}^{ \pm}$into Eq. (B.5), further making use of Eq. (B.4) to express the link-wise operators in terms of FD stencils, leads us to:

$$
\begin{aligned}
\bar{\rho} a_{x} \delta t 3 \sum_{q=1}^{Q-1} t_{q}^{\star} \frac{c_{q x}^{2} c_{q y}^{2}}{c^{4}}= & \bar{\Delta}_{y} P \delta t \sum_{q=1}^{Q-1} t_{q}^{\star} \frac{c_{q x} c_{q y}}{c^{2}} \\
& +\bar{\Delta}_{y}(\bar{\rho} u v) \delta t \frac{6}{2} \sum_{q=1}^{Q-1} t_{q}^{\star} \frac{c_{q x}^{2} c_{q y}^{2}}{c^{4}} \\
& +\mathcal{A} \Lambda^{+} \delta t^{2} \bar{\Delta}_{y}\left(\bar{\rho} a_{x} v+\bar{\rho} a_{y} u\right) 3 \sum_{q=1}^{Q-1} t_{q}^{\star} \frac{c_{q x}^{2} c_{q y}^{2}}{c^{4}} \\
& -\Lambda^{+} \delta t^{2} \bar{\Delta}_{y}^{2}(\bar{\rho} u) \sum_{q=1}^{Q-1} t_{q}^{\star} \frac{c_{q x}^{2} c_{q y}^{2}}{c^{2}} \\
& -\Lambda \delta t^{3} \bar{\Delta}_{y}^{2}\left(\bar{\rho} a_{x}\right) \sum_{q=1}^{Q-1} t_{q}^{\star} \frac{c_{q x}^{2} c_{q y}^{2}}{c^{2}} \\
& +\left(\Lambda-\frac{1}{4}\right) \delta t^{3} 3 \bar{\Delta}_{y}^{2}\left(\bar{\rho} a_{x}\right) \sum_{q=1}^{Q-1} t_{q}^{\star} \frac{c_{q x}^{2} c_{q y}^{4}}{c^{4}} .
\end{aligned}
$$

For standard lattices, the second- and fourth-order velocity moments are $\sum_{q=1}^{Q-1} t_{q}^{\star} c_{q \alpha} c_{q \beta}=\delta_{\alpha \beta} c^{2}, \forall \alpha, \beta$, and $\sum_{q=1}^{Q-1} t_{q}^{\star} c_{q \alpha}^{2} c_{q \beta}^{2}=$ $\frac{1}{3} c^{4}$, with $\alpha \neq \beta$. The sixth-order moment is anisotropic. In a horizontal setup, it yields $\sum_{q=1}^{Q-1} t_{q}^{\star} c_{q \alpha}^{2} c_{q \beta}^{4}=\frac{1}{3} c^{6}$ with $\alpha \neq \beta$. After computing each velocity moment in Eq. (B.8) we obtain:

$$
\begin{aligned}
\bar{\rho} a_{x}= & \bar{\Delta}_{y}(\bar{\rho} u v)+\mathcal{A} \Lambda^{+} \delta t \bar{\Delta}_{y}\left(\bar{\rho} a_{x} v+\bar{\rho} a_{y} u\right)-\frac{\Lambda^{+}}{3} c^{2} \delta t \bar{\Delta}_{y}^{2}(\bar{\rho} u) \\
& -\frac{\Lambda}{3} c^{2} \delta t^{2} \bar{\Delta}_{y}^{2}\left(\bar{\rho} a_{x}\right)+\left(\Lambda-\frac{1}{4}\right) c^{2} \delta t^{2} \bar{\Delta}_{y}^{2}\left(\bar{\rho} a_{x}\right) .
\end{aligned}
$$

After re-arranging Eq. (B.9) and expressing the FD stencils according to Eq. (B.4), with $\delta x=c \delta t$ considered, we obtain:

$$
\begin{aligned}
& \frac{u_{j+1} v_{j+1}-u_{j-1} v_{j-1}}{2 \delta x}-\left(a_{x}\right)_{j}-\frac{\Lambda^{+}}{3} c^{2} \delta t \frac{u_{j+1}-2 u_{j}+u_{j-1}}{\delta x^{2}} \\
& +\delta x^{2}\left(\frac{8 \Lambda-3}{12}\right) \frac{\left(a_{x}\right)_{j+1}-2\left(a_{x}\right)_{j}+\left(a_{x}\right)_{j-1}}{\delta x^{2}} \\
& +\mathrm{A} \Lambda^{+} \delta t\left(\frac{u_{j+1}\left(a_{y}\right)_{j+1}-u_{j-1}\left(a_{y}\right)_{j-1}}{2 \delta x}+\frac{v_{j+1}\left(a_{x}\right)_{j+1}-v_{j-1}\left(a_{x}\right)_{j-1}}{2 \delta x}\right)=0 .
\end{aligned}
$$

Then, with $\Lambda^{+}=\left(\tau^{+}-1 / 2\right)$, we finally arrive at Eq. (8).

Discussion: The end result deduced here in Appendix $B$ is identical to the analytical solution obtained in Appendix A. However, compared to the He et al. [46] derivation summarized in Appendix A, the procedure presented here considerably simplifies the workload required. Most importantly, Ginzburg and d'HumiÃlires procedure [14,47] offers a rather more general framework to access the LBM solution. The reason is that it solves for equilibrium and non-equilibrium components of populations separately. Thus, it is easily applicable/extendable to other types of 
analyses, such as in the study of boundary schemes or interfaces $[14,32,33]$.

\section{References}

[1] Frisch U, d'Humières D, Hasslacher B, Lallemand $P$, Pomeau $Y$, Rivet JP. Lattice gas hydrodynamics in two and three dimensions. Complex Syst 1987; 1:649-707.

[2] Higuera FJ, Jiménez J. Boltzmann approach to lattice gas simulations. Europhys Lett 1989;9:663-8.

[3] Higuera FJ, Succi S, Benzi R. Lattice gas dynamics with enhanced collisions. Europhys Lett 1989;9:345-9.

[4] Chen S, Doolen G. Lattice Boltzmann method for fluid flows. Ann Rev Fluid Mech 1998;30:329-64.

[5] Aidun CK, Clausen JR. Lattice-Boltzmann method for complex flows. Ann Rev Fluid Mech 2010;42:439-72.

[6] Succi S. The lattice Boltzmann equation: for fluid dynamics and beyond. Oxford University Press; 2001.

[7] Krüger T, Kusumaatmaja H, Kuzmin A, Shardt O, Silva G, Viggen EM. The lattice Boltzmann method - principles and practice. 1st ed. Springer International Publishing; 2016.

[8] Wagner AJ. Thermodynamic consistency of liquid-gas lattice Boltzmann simulations. Phys Rev E 2006;74:056703.

[9] Huang H, Krafczyk M, Lu M. Forcing term in single-phase and Shan-Chen-type multiphase lattice Boltzmann models. Phys Rev E 2011;84:046710.

[10] Guo Z, Zheng C, Shi B. Force imbalance in lattice Boltzmann equation for two-phase flows. Phys Rev E 2011;83:036707.

[11] Xiong Y, Guo Z. Effects of density and force discretizations on spurious velocities in lattice Boltzmann equation for two-phase flows. J Phys A 2014; 47:195502.

[12] Guo Z, Zhao TS. Lattice Boltzmann model for incompressible flows through porous media. Phys Rev E 2002;66:036304.

[13] Nie X, Martys NS. Breakdown of Chapman-Enskog expansion and the anisotropic effect for Lattice-Boltzmann models of porous flow. Phys Fluids 2007; 19:011702.

[14] Ginzburg I. Consistent lattice Boltzmann schemes for the Brinkman model of porous flow and infinite Chapman-Enskog expansion. Phys Rev E 2008;77:66704.

[15] Liu Q He YL. Multiple-relaxation-time lattice Boltzmann modeling of incompressible flows in porous media. Physica A 2015;429:215-30.

[16] Salmon R. The lattice Boltzmann method as a basis for ocean circulation modeling. J Marine Res 1999;57:503-35.

[17] Dellar P.J.. A priori derivation of lattice Boltzmann equations for rotating fluids. Preprint.

[18] Zhou D, Lu Z, Guo T. A rotating reference frame based lattice Boltzmann flux solver for simulation of turbomachinery flows. Int J Numer Methods Fluids 2017;83:561-82.

[19] Liou T-M, Wang CS. Large eddy simulation of rotating turbulent flows and heat transfer by the lattice Boltzmann method. Phys Fluids 2018;30:015106.

[20] Premnath KN, Pattison MJ, Banerjee S. Generalized lattice Boltzmann equation with forcing term for computation of wall-bounded turbulent flows. Phys Rev E 2009;79:026703.

[21] Kareema WA, Izawab S, Xiongc A-K, Fukunishib Y. Lattice Boltzmann simulations of homogeneous isotropic turbulence. Comput Math Appl 2009;58:1055-66.

[22] Jahanshaloo L, Pouryazdanpanah E, Sidik NAC. A review on the application of the lattice Boltzmann method for turbulent flow simulation. Numer Heat Transf Part A 2013;64:938.

[23] Haussmann M, Simonis S, Nirschl N, Krause MJ. Direct numerical simulation of decaying homogeneous isotropic turbulence numerical experiments on stability, consistency and accuracy of distinct lattice Boltzmann methods. Int J Mod Phys C 2019;30:1950074.

[24] Luo LS. Unified theory of lattice Boltzmann models for nonideal gases. Phys Rev Lett 1998;81:1618.

[25] Martys NS, Shan X, Chen H. Evaluation of the external force term in the discrete boltzmann equation. Phys Rev E 1998;58:6855.
[26] Shan X, Yuan X-F, Chen H. Kinetic theory representation of hydrodynamics: a way beyond the Navier-Stokes equation. J Fluid Mech 2006;550:413.

[27] Ginzbourg I, Adler PM. Boundary flow condition analysis for the three-dimensional lattice Boltzmann model. J Phys II 1994;4:191.

[28] Ladd AJC. Numerical simulations of particulate suspensions via a discretized Boltzmann equation. Part 1. Theoretical foundation. J Fluid Mech 1994;271:285

[29] Buick JM, Greated CA. Gravity in a lattice Boltzmann model. Phys Rev E 2000;61:5307.

[30] Guo Z, Zheng C, Shi B. Discrete lattice effects on the forcing term in the lattice Boltzmann method. Phys Rev E 2002;65:046308.

[31] Nash RW, Adhikari R, Cates ME. Singular forces and pointlike colloids in lattice Boltzmann hydrodynamics. Phys Rev E 2008;77:026709.

[32] Ginzburg I, Silva G, Talon L. Analysis and improvement of Brinkman lattice Boltzmann schemes: bulk, boundary, interface. Similarity and distinctness with finite elements in heterogeneous porous media. Phys Rev E 2015;91:23307.

[33] Silva G, Talon L, Ginzburg I. Low-and high-order accurate boundary conditions: from Stokes to Darcy porous flow modeled with standard and improved Brinkman lattice Boltzmann schemes. J Comput Phys 2017;335:50-83.

[34] Silva G, Ginzburg I. The permeability and quality of velocity field in a square array of solid and permeable cylindrical obstacles with the TRT-LBM and FEM Brinkman schemes. Comptes Rendus Mécanique 2015;343:545-58.

[35] Guo Z, Zheng C, Shi B. Incompressible lattice Boltzmann model for porous flows with large pressure gradient. Prog Compu Fluid Dy 2008;9:225.

[36] Lycett-Brown D, Luo KH. Improved forcing scheme in pseudopotential lattice Boltzmann methods for multiphase flow at arbitrarily high density ratios. Phys Rev E 2015;91:023305.

[37] Ginzburg I, Verhaeghe F, d'Humières D. Two-relaxation-time lattice Boltzman scheme: about parametrization, velocity, pressure and mixed conditions. Commun Comp Phys 2008;3:427.

[38] Silva G. Semiao V. A study on the inclusion of body forces in the lattice Boltzmann BGK equation to recover steady-state hydrodynamics. Physica A 2011;390:1085-95.

[39] Silva G, Semiao V. First- and second-order forcing expansions in a lattice Boltzmann method reproducing isothermal hydrodynamics in artificial compressibility form. J Fluid Mech 2012;698:282-303.

[40] Junk M, Klar A, Luo LS. Asymptotic analysis of the lattice Boltzmann equation. J Stat Phys 2005;210:676-704

[41] Junk M, Yang Z. Analysis and invariant properties of a lattice Boltzmann method. Adv Appl Math Mech 2010;5:640-69.

[42] Fletcher C. Computational techniques for fluid dynamics 1. 2nd ed. Berlin Heidelberg: Springer-Verlag; 1998.

[43] Chopard B, Falcone JL, Latt J. The lattice Boltzmann advection-diffusion model revisited. Eur Phys J Spec Top 2009;171:245-9.

[44] Ginzburg I. Truncation errors, exact and heuristic stability analysis of two-relaxation-times lattice Boltzmann schemes for anisotropic advection-diffusion equation. Commun Comput Phys 2012;11:1439-502.

[45] Ginzburg I. Truncation effect on Taylor-Aris dispersion in lattice Boltzmann schemes: accuracy towards stability. J Comput Phys 2015;299:974-1003.

[46] He X, Zou Q, Luo L-S, Dembo M. Analytical solutions of simple flows and analysis of nonslip boundary conditions for the lattice Boltzmann BGK model. J Stat Phys 1997;87:115-36.

[47] d'Humières D, Ginzburg I. Viscosity independent numerical errors for lattice Boltzmann models: from recurrence equations to "magic" collision numbers. Comput Math Appl 2009;58:823.

[48] Le G, Zhang J. Boundary slip from the immersed boundary lattice Boltzmann models. Phys Rev E 2009;79:026701.

[49] He X, Zou Q, Luo L-S, Dembo M. Lattice Boltzmann model for the incompressible Navier-Stokes equation. I Stat Phys 1997:88:927-44.

[50] Qian Y, d'Humières D, Lallemand P. Lattice BGK models for Navier-Stokes equation. Europhys Lett 1992;17:479-83.

[51] Zou Q, He X. On pressure and velocity boundary conditions for the lattice Boltzmann BGK model. Phys Fluids 1997;9:1592-8.

[52] Postma B., Silva G.. Force methods for the two-relaxation-times lattice Boltzmann. Under preparation. 